\title{
Dynamic contracting with limited commitment and the ratchet effect
}

\author{
Dino Gerardi \\ Collegio Carlo Alberto, Università di Torino \\ LuCAs MAestri \\ EPGE, Escola Brasileira de Economia e Finanças
}

\begin{abstract}
We study dynamic contracting with adverse selection and limited commitment. A firm (the principal) and a worker (the agent) interact for potentially infinitely many periods. The worker is privately informed about his productivity and the firm can only commit to short-term contracts. The ratchet effect is in place since the firm has the incentive to change the terms of trade and offer more demanding contracts when it learns that the worker is highly productive.

As the parties become arbitrarily patient, the equilibrium outcome takes one of two forms. If the prior probability of the worker being productive is low, the firm offers a pooling contract and no information is ever revealed. In contrast, if this prior probability is high, the firm fires the unproductive worker at the beginning of the relationship.
\end{abstract}

KeYwords. Dynamic contracting, limited commitment, ratchet effect.

JEL CLASSification. D80, D82, D86.

\section{INTRODUCTION}

This paper contributes to the literature on the ratchet effect by analyzing an infinite horizon contracting problem with short-term contracts. We frame the analysis in the context of a labor relationship between a worker and a firm. In each period, the worker can produce a good of quality $q \in[0,1]$ at a cost that is linear in $q$. The worker is privately informed about his (persistent) marginal cost, which is low with prior probability $p_{0}$ and high with probability $1-p_{0}$. The firm can only commit to short-term contracts, which indicate the payment that the worker receives in the current period if he produces a good of a specified quality. In each period in which the worker is employed, the firm

Dino Gerardi: dino.gerardi@carloalberto.org

Lucas Maestri: lucas . maestri@fgv.br

We are grateful to the three anonymous referees, Luca Anderlini, Paolo Ghirardato, Daniel Gottlieb, Felipe Iachan, Ignacio Monzón, Juan Morales, Cézar Santos, and various seminar audiences for useful comments. The financial support of the European Research Council (Consolidator Grant INFOASYMMARKETS) is gratefully acknowledged. Lucas Maestri acknowledges that this study was financed in part by the Coordenação de Aperfeiçoamento de Pessoal de Nível Superior, Brasil (CAPES), finance code 001. Lucas Maestri is also grateful for the financial support of CNPq-National Council for Scientific and Technological Development.

(C) 2020 The Authors. Licensed under the Creative Commons Attribution-NonCommercial License 4.0. Available at https://econtheory.org. https://doi.org/10.3982/TE2449 
offers a menu with finitely many contracts. The worker can either accept one contract in the menu or reject all contracts and end the relationship.

We show that when the discount factor is not too high, the firm is able to extract the worker's private information independently of the value of the prior. In particular, if the prior $p_{0}$ is high, the firm offers a firing menu in every period. A firing menu contains only one contract, which specifies the efficient quality when the cost is low and yields a payoff equal to 0 to the worker. The contract is only accepted by the low-cost worker. Thus, the firm learns the worker's cost in the first period by firing the high-cost worker.

When the prior $p_{0}$ is not too high, the firm employs a sequentially screening procedure. The firm offers two contracts until it discovers the worker's cost, which occurs in finitely many periods. During the screening procedure, the high-cost worker accepts the first contract while the low-cost worker randomizes between the two contracts. Once the screening process is complete and the firm discovers the cost, the worker delivers the efficient quality and obtains a 0 payoff.

When the parties are sufficiently patient, the sequentially screening procedure is infeasible. Consider the last period of the screening procedure in which the firm offers a menu that fully separates the two types of worker (in the sense that each type accepts a different contract). The low-cost worker can guarantee a large future payoff by mimicking the high-cost worker. Because of this, it is impossible to design two contracts that simultaneously satisfy the truthtelling constraints of the two types of worker. Only a sufficiently generous contract can prevent the low-cost worker from imitating the highcost worker. But in this case, the high-cost worker has an incentive to adopt the "take the money and run" strategy (i.e., accept the contract designed for the low-cost worker and then quit the relationship). ${ }^{1}$

The firm could, in principle, adopt more complex dynamic screening strategies. For instance, in the initial phase of the relationship, both types of worker could accept different contracts in the menu with positive, but different, probabilities. Then the firm could use the available information to induce partial or even complete separation (through a firing menu). To investigate the feasibility and optimality of such strategies, we analyze the limiting outcome, as the parties become arbitrarily patient, of all perfect Bayesian equilibria.

We show that the limiting equilibrium outcome is unique and takes a very simple form. If the prior is below a certain threshold $\hat{p}$, then, in every period, the firm offers the most profitable contract that the high-cost worker is willing to accept. Both types of worker accept the contract (i.e., they pool) and there is no learning. In contrast, if the prior is above $\hat{p}$, the firm offers the firing menu and the high-cost worker quits the relationship without delay. In both cases, the limiting equilibrium allocation is inefficient.

Our results illustrate that when the parties are sufficiently patient, the firm can only screen the worker's type by firing the high-cost worker. The driving forces behind our findings are similar to those that prevent full separation. When the discount factor is large, it is very costly for the firm to separate the two types of worker and continue the relationship with both of them. A lasting relationship with the high-cost worker provides

\footnotetext{
${ }^{1}$ A similar result appears in Laffont and Tirole (1990).
} 
strong incentives to the low-cost worker to misrepresent his information. Using this fact, we show that the firm would not benefit from engaging in partial screening strategies, even if such policies were feasible.

Our benchmark model assumes that the relationship ends when the worker rejects all the contracts in the firm's menu. This modeling assumption captures situations in which the parties are committed to terminate the relationship upon disagreement. Of course, one can also imagine situations in which the relationship continues even when the parties do not reach an agreement. Therefore, we analyze an extension of the model that allows for rehiring. We study the infinitely repeated game in which, in each period, the firm proposes a menu of contracts from which the worker has to select at most one. We first show that the complete-information version of this game admits a folk theorem. Although the firm has the bargaining power to make offers, the worker can obtain large payoffs by rejecting unfavorable contracts. This is possible because the acceptance of unfavorable contracts by the worker triggers a continuation equilibrium in which the firm implements an efficient allocation that yields a zero payoff to the worker. We use these findings from the complete-information game to show that a version of the folk theorem holds for our model with rehiring. ${ }^{2}$ In particular, when the parties are sufficiently patient, the firm can obtain a payoff arbitrarily close to the payoff of the optimal mechanism with commitment.

This paper contributes to the literature on repeated adverse selection with limited commitment pioneered by Freixas et al. (1985), Gibbons (1987), and Laffont and Tirole $(1987,1988)$. In these seminal papers, the parties interact for two periods. One of the main findings is that there is partial separation of the agent's types in the first period (i.e., the equilibrium is semipooling) and full separation in the second and final period. Therefore, the outcome of two-period environments presents gradual information revelation. In contrast, our paper shows that when the relationship is infinitely repeated and the prior is low, the equilibrium allocation is close to a pooling allocation when the parties are patient.

Hart and Tirole (1988) analyze a dynamic model in which the seller makes a rental offer to the buyer in every period. The buyer's valuation for the good is private information and can take on two values, both of which are larger than the seller's cost of producing the good. As the parties become sufficiently patient, the equilibrium allocation converges to the efficient allocation, in which both types of buyers consume the good in every period. Note that for high values of the prior, this pooling allocation coincides with the seller's optimal mechanism under full commitment (i.e., lack of commitment is not detrimental to the seller's payoff). In a recent paper, Beccuti and Möller (2018) extend Hart and Tirole's analysis to the case in which the seller is more patient than the buyer. Halac (2012) studies a relational contract model in which the principal is privately informed about his outside option. When the uninformed party has the bargaining power, Coasian forces lead to a pooling outcome when the parties are sufficiently patient. Our work differs from these papers in two respects. First, in our model, the agent's private

\footnotetext{
${ }^{2}$ This finding is reminiscent of earlier contributions to repeated games with incomplete information and simultaneous moves (see Peski 2008 and the references therein).
} 
information is necessary to determine the best course of action and, therefore, pooling allocations are never optimal for the firm under full commitment. Second, we analyze environments in which the ratchet effect leads to inefficiencies. ${ }^{3}$

Our work is also related to the literature on renegotiation. The seminal paper by Laffont and Tirole (1990) analyzes a two-period model. Recently, Strulovici (2017) and Maestri (2017) study renegotiation in infinite horizon models. These studies find that equilibrium allocations become efficient as the parties become arbitrarily patient. In contrast, in our model the limit allocation is inefficient whenever the firing allocation is not a commitment solution.

Bhaskar (2014) studies learning in a dynamic model in which the principal and the agent are ex ante symmetrically informed about the job's difficulty. When the agent's effort is unobservable, it is impossible for the principal to design a contract that induces an interior effort level in the first period. Bhaskar and Mailath (2019) consider a related dynamic model and show that inducing high effort becomes prohibitively costly for the principal as the parties become arbitrarily patient. Therefore, the ratchet effect imposes stringent constraints on the learning process of the relationship. In contrast, our paper assumes adverse selection and no exogenous learning, and concludes that the ratchet effect imposes constraints on the amount of private information that is revealed in a dynamic relationship.

There is also a connection between our paper and the literature on durable goods monopoly under limited commitment. Ausubel and Deneckere (1989) study a model in which the seller posts prices and obtain a folk theorem for the "no gap" case. In our context, a folk theorem holds when rehiring is possible. Skreta $(2006,2015)$ analyzes more general selling mechanisms and shows that posting a price is the seller's optimal strategy. In these studies, the relationship between the buyer and the seller ends as soon as the durable good is traded, while in our model the parties can make a new transaction every period.

Finally, a number of authors have identified situations in which the ratchet effect is mitigated. Kanemoto and MacLeod (1992) argue that competition for secondhand workers guarantees the existence of efficient piece-rate contracts in long-term relationships. Carmichael and MacLeod (2000) show that if entry in a market is difficult, then it is possible to sustain cooperation between an infinitely lived firm and a stream of short lived workers. Our findings suggest that rehiring is another possible remedy to the ratchet effect.

The rest of the paper is organized as follows. We present the model in Section 2. In Section 3, we briefly discuss the mechanism design problem with commitment. In Section 4, we show existence of equilibria and provide conditions under which all private information is revealed. Section 5 contains the main result, which completely characterizes the unique equilibrium outcome when the parties are arbitrarily patient. In Section 6, we analyze the extension of the model in which rehiring is possible. Section 7 concludes. Most proofs are relegated to a number of appendices and

\footnotetext{
${ }^{3}$ Our work analyzes the relationship between two infinitely lived players. In the context of political economy, several papers study the effects of limited commitment in repeated interactions between one principal and a continuum of privately informed agents (see, among others, Acemoglu et al. 2010, Farhi et al. 2012, and Scheuer and Wolitzky 2016).
} 
the Supplemental Material, available in a supplementary file on the journal website, http://econtheory.org/supp/2449/supplement.pdf.

\section{THE MODEL}

We study a dynamic principal-agent model with adverse selection and short-term contracts, framed in the context of a labor relationship between a firm and a worker.

The worker is privately informed about his (persistent) type, which is equal to $L$ with prior probability $p_{0} \in(0,1)$ and is equal to $H$ with probability $1-p_{0}$. The firm and the worker interact for potentially infinitely many periods. In each period, the worker of type $i \in\{H, L\}$ can produce a good of quality $q \in[0,1]$ at a cost of $\theta_{i} q$, where $0<$ $\theta_{L}<\theta_{H}$. We refer to the low type $L$ (high type $H$ ) as the low- (high-) cost worker. We write $\Delta \theta:=\theta_{H}-\theta_{L}$. The worker bears an additional cost $\alpha>0$ in every period in which he interacts with the firm. The cost $\alpha$ can be interpreted as the per-period payoff of an outside option available to the worker if he ends the relationship (in Section 7, we discuss the case $\alpha=0$ ).

The firm's valuation of a good of quality $q$ is $v(q)$. The function $v:[0,1] \rightarrow \mathbb{R}_{+}$is twice continuously differentiable, increasing, strictly concave, and satisfies $v(0)=0 .{ }^{4}$

Both parties' preferences are linear in money. When the worker produces a good of quality $q$ and the firm makes a transfer equal to $x$, the payoff of type $i \in\{H, L\}$ is $x-\theta_{i} q-\alpha$, while the firm's payoff is $v(q)-x$.

We let $q_{i}^{*}, i \in\{H, L\}$, denote the efficient quality produced by type $i$ :

$$
q_{i}^{*}=\underset{q \in[0,1]}{\arg \max }\left(v(q)-\theta_{i} q\right) .
$$

To make the problem interesting, we assume

$$
v\left(q_{H}^{*}\right)-\theta_{H} q_{H}^{*}-\alpha>0 .
$$

This assumption guarantees that the firm prefers hiring the high-cost worker over collecting its outside option, which yields a payoff equal to 0 . Moreover, we assume that $q_{H}^{*} \in(0,1)$ and, therefore, $q_{L}^{*}>q_{H}^{*} \cdot{ }^{5}$ In this case, the efficient allocation varies with worker's type.

The firm and the worker play the following game. At the beginning of period $t=$ $0,1, \ldots$, the firm offers a menu $m_{t}$ of contracts to the worker. Each contract is of the form $\left(x_{t}, q_{t}\right)$ and specifies the transfer $x_{t}$ paid by the firm and the quality $q_{t} \in[0,1]$ that the worker must produce. We assume that the quality is verifiable and, thus, each contract is enforceable. After receiving the menu $m_{t}$, the worker has two options: (i) selecting a

\footnotetext{
${ }^{4}$ The concavity of $v(\cdot)$ guarantees that the firm's screening problem in the proof of Proposition 1 is well behaved. The concavity also allows us to derive a number of useful bounds in the proof of Proposition 2 . Finally, the assumption $v^{\prime}(0)<\infty$ implies that for large values of the prior, the solution to the mechanism design problem with commitment is to fire the high type (see Section 3). This is used in the proof of the main result.

${ }^{5}$ In particular, we use this assumption in the proof of Proposition 1 to construct a sequence of separating contracts.
} 
contract from the menu or (ii) rejecting all the contracts and quitting the relationship. In the first case, the game moves to the next period $t+1$. In the second case, the game ends and both parties obtain a continuation payoff equal to 0 . The parties discount future payoffs at the common discount factor $\delta \in(0,1)$.

We let $\mathcal{M}=\bigcup_{j=1}^{M}(\mathbb{R} \times[0,1])^{j}$ denote the set of available menus, where $M \in\{2,3, \ldots\}$ is an exogenous upper bound to the number of contracts that a menu can contain. The restriction $M \geq 2$ guarantees that the menus can contain two contracts (so that it is possible for the firm to separate the two worker types). When the firm offers the menu $m_{t}$, the set of actions available to the worker is $m_{t} \cup\{\varnothing\}$, where $\varnothing$ denotes the choice of rejecting all the contracts in $m_{t}$ and quitting. We let $a_{t}$ denote the agent's decision in period $t$.

For every $t=1,2, \ldots$, a period- $t$ (nonfinal) public history $h^{t}=\left(m_{0}, a_{0}, \ldots, m_{t-1}, a_{t-1}\right)$ consists of all the menus offered by the firm in the previous periods $\tau=0, \ldots, t-1$, as well as all the worker's decisions, provided that he never chose to quit (i.e., $a_{\tau} \neq \varnothing$ for every $\tau=0, \ldots, t-1)$. We let $H^{0}=\left\{h^{0}\right\}$ denote the set containing the empty history $h^{0}$. We write $H^{t}$ for the set of all period- $t$ public histories. Finally, $\mathcal{H}=\bigcup_{t=0,1, \ldots} H^{t}$ is the set of all (nonfinal) public histories.

A behavior strategy $\sigma^{F}$ for the firm is a sequence $\left\{\sigma_{t}^{F}\right\}$, where $\sigma_{t}^{F}$ is a function from $H^{t}$ into $\Delta(\mathcal{M})$, mapping the history $h^{t}$ into a (possibly random) menu. A behavior strategy $\left(\sigma^{H}, \sigma^{L}\right)$ for the worker is a sequence $\left\{\left(\sigma_{t}^{H}, \sigma_{t}^{L}\right)\right\}$, where $\sigma_{t}^{i}, i \in\{H, L\}$, associates to every pair $\left(h^{t}, m_{t}\right) \in H^{t} \times \mathcal{M}$ a probability distribution over the set $m_{t} \cup\{\varnothing\}$. We write $\sigma=\left(\sigma^{F}, \sigma^{H}, \sigma^{L}\right)$ for a strategy profile. Finally, we let $\mu=\left\{\mu\left(h^{t}\right), \mu\left(h^{t}, m_{t}\right)\right\}_{h^{t} \in \mathcal{H}, m_{t} \in \mathcal{M}}$ denote the firm's system of beliefs, where $\mu\left(h^{t}\right)$ and $\mu\left(h^{t}, m_{t}\right)$ represent the probability that the firm assigns, at the history $h^{t}$ and $\left(h^{t}, m_{t}\right)$, respectively, to the event that the worker's type is equal to $L$.

Our solution concept is perfect Bayesian equilibrium (PBE or equilibrium henceforth), formally defined below.

Definition 1. A PBE of our game is a strategy profile $\sigma$ and a system of beliefs $\mu$ such that the following statements hold:

(i) The strategy profile $\sigma$ is sequentially rational given $\mu$.

(ii) For every history $\left(h^{t}, m_{t}\right) \in H^{t} \times \mathcal{M}, \mu\left(h^{t}, m_{t}\right)=\mu\left(h^{t}\right)$.

(iii) For every history $\left(h^{t}, m_{t}\right) \in H^{t} \times \mathcal{M}$ and for every action $a_{t} \in m_{t} \cup\{\varnothing\}$, if

$$
\left(1-\mu\left(h^{t}\right)\right) \sigma_{t}^{H}\left(a_{t} \mid h^{t}, m_{t}\right)+\mu\left(h^{t}\right) \sigma_{t}^{L}\left(a_{t} \mid h^{t}, m_{t}\right)>0,
$$

then the belief $\mu\left(h^{t}, m_{t}, a_{t}\right)$ is derived from $\mu\left(h^{t}\right)$ according to Bayes' rule:

$$
\mu\left(h^{t}, m_{t}, a_{t}\right)=\frac{\mu\left(h^{t}\right) \sigma_{t}^{L}\left(a_{t} \mid h^{t}, m_{t}\right)}{\left(1-\mu\left(h^{t}\right)\right) \sigma_{t}^{H}\left(a_{t} \mid h^{t}, m_{t}\right)+\mu\left(h^{t}\right) \sigma_{t}^{L}\left(a_{t} \mid h^{t}, m_{t}\right)} .
$$

In addition to sequential rationality and Bayesian updating whenever possible (i.e., including off-path histories $\left(h^{t}, m_{t}, a_{t}\right)$ that are reached with positive probability given 
$\left.\left(h^{t}, m_{t}\right)\right)$, the concept of PBE imposes the "no signaling what you don't know" condition (Fudenberg and Tirole (1991)) in the sense that the firm does not revise its belief after proposing a menu.

Given a strategy profile $\sigma$ and a system of beliefs $\mu$, for each history $h^{t}$, we let $V_{F}\left(h^{t} ;(\sigma, \mu)\right)$ denote the firm's continuation payoff at $h^{t}$. We also let $\mathbb{T} \in \mathbf{N} \cup\{\infty\}$ denote the random period in which the relationship terminates (we set $\mathbb{T}=\infty$ if the worker remains employed forever). ${ }^{6}$ Then we have

$$
V_{F}\left(h^{t} ;(\sigma, \mu)\right):=\mathbb{E}_{(\sigma, \mu)}\left[(1-\delta) \sum_{\tau=t}^{\mathbb{T}-1} \delta^{\tau-t}\left(v\left(q_{\tau}\right)-x_{\tau}\right) \mid h^{t}\right],
$$

where $\mathbb{E}_{(\sigma, \mu)}\left[Y \mid h^{t}\right]$ represents the conditional expected value (given $h^{t}$ ) of the random variable $Y$ given the strategy profile $\sigma$ and the system of beliefs $\mu$. Analogously, for every history $h^{t}$ we let $W_{i}\left(h^{t} ;(\sigma, \mu)\right)$ denote the expected continuation payoff at $h^{t}$ of the worker of type $i \in\{H, L\}$. We have

$$
W_{i}\left(h^{t} ;(\sigma, \mu)\right):=\mathbb{E}_{(\sigma, \mu)}\left[(1-\delta) \sum_{\tau=t}^{\mathbb{T}-1} \delta^{\tau-t}\left(x_{\tau}-\theta_{i} q_{\tau}-\alpha\right) \mid i, h^{t}\right] .
$$

To simplify the notation, we omit the argument $(\sigma, \mu)$ and write $V_{F}\left(h^{t}\right)$ and $W_{i}\left(h^{t}\right)$ when there is no ambiguity. We also use $V_{F}\left(h^{t}, m_{t}\right)$ and $W_{i}\left(h^{t}, m_{t}\right), i \in\{H, L\}$, to denote the firm's and worker's payoff at the history $\left(h_{t}, m_{t}\right)$.

For $i \in\{H, L\}$ and $q \in[0,1]$, we let

$$
\pi_{i}(q):=v(q)-\theta_{i} q-\alpha
$$

denote the firm's profits when the quality is $q$, the worker is of type $i$, and the firm pays the reservation wage $\theta_{i} q+\alpha$. Therefore, $\pi_{i}\left(q_{i}^{*}\right)$ represents the highest level of profits that the firm can achieve from the interaction with type $i$. As $\pi_{L}\left(q_{L}^{*}\right)>\pi_{H}\left(q_{H}^{*}\right)$, let $\hat{p} \in(0,1)$ be defined by $\pi_{H}\left(q_{H}^{*}\right)=\hat{p} \pi_{L}\left(q_{L}^{*}\right)$.

We conclude this section with a simple result that provides a lower bound to the firm's payoff under any PBE.

Lemma 1. Fix a $\operatorname{PBE}(\sigma, \mu)$. For every history $h^{t} \in \mathcal{H}$, we have

$$
V_{F}\left(h^{t} ;(\sigma, \mu)\right) \geq \max \left\{\pi_{H}\left(q_{H}^{*}\right), \mu\left(h^{t}\right) \pi_{L}\left(q_{L}^{*}\right)\right\} .
$$

Proof. By contradiction, suppose that there exist a PBE $(\sigma, \mu)$, a history $h^{t}$, and $\varepsilon>0$ such that

$$
V_{F}\left(h^{t} ;(\sigma, \mu)\right)<\max \left\{\pi_{H}\left(q_{H}^{*}\right), \mu\left(h^{t}\right) \pi_{L}\left(q_{L}^{*}\right)\right\}-\varepsilon .
$$

Suppose that $\pi_{H}\left(q_{H}^{*}\right)>\mu\left(h^{t}\right) \pi_{L}\left(q_{L}^{*}\right)$. If the firm offers the menu $\left\{\left(\theta_{H} q_{H}^{*}+\alpha+\right.\right.$ $\left.\left.\frac{\varepsilon}{2}, q_{H}^{*}\right)\right\}$ in every period $t, t+1, \ldots$ (notice that both types strictly prefer to accept the

\footnotetext{
${ }^{6}$ Here, and in what follows, we use $\mathbf{N}=\{0,1, \ldots\}$ to denote the set of nonnegative integers and adopt the convention that $\sum_{\tau=t}^{t-1} \delta^{\tau-t}=0$.
} 
contract in the menu rather than quit the relationship), then its continuation payoff is

$$
\pi_{H}\left(q_{H}^{*}\right)-\frac{\varepsilon}{2}>V_{F}\left(h^{t} ;(\sigma, \mu)\right)
$$

which is a contradiction.

Next consider the case $\pi_{H}\left(q_{H}^{*}\right) \leq \mu\left(h^{t}\right) \pi_{L}\left(q_{L}^{*}\right)$. The firm can guarantee a continuation payoff at least equal to

$$
\mu\left(h^{t}\right)\left(\pi_{L}\left(q_{L}^{*}\right)-\frac{\varepsilon}{2}\right)>V_{F}\left(h^{t} ;(\sigma, \mu)\right)
$$

by offering the menu $\left\{\left(\theta_{L} q_{L}^{*}+\alpha+\frac{\varepsilon}{2}, q_{L}^{*}\right)\right\}$ in every period $t, t+1, \ldots$ (in equilibrium, the low type must accept the contract in the menu).

Intuitively, the following two options are always available to the firm. The first option is to stop learning and offer $\left(\theta_{H} q_{H}^{*}+\alpha, q_{H}^{*}\right)$, the most profitable contract in the class of contracts that are accepted by both types of worker. The second option is to fire the highcost worker and interact only with the low-cost worker. In this case, the most profitable contract is $\left(\theta_{L} q_{L}^{*}+\alpha, q_{L}^{*}\right)$.

\section{THE COMMITMENT ALLOCATION}

It is useful to review the benchmark model in which the firm can fully commit to a sequence of menus $\left(m_{0}, m_{1}, \ldots\right)$. This benchmark provides an upper bound to the firm's profits in the game with limited commitment. It is well known that the solution to the firm's commitment problem is to replicate the optimal static mechanism (see, for example, Chapter 1 in Laffont and Tirole 1993).

The optimal static mechanism is as follows. There exists a critical value $p^{C} \in(\hat{p}, 1)$ such that if $p_{0}>p^{C}$, the optimal menu (with commitment) is unique and equal to $\left\{\left(\theta_{L} q_{L}^{*}+\alpha, q_{L}^{*}\right)\right\} .^{7}$ The low-cost worker accepts the contract in the menu while the highcost worker rejects it. Thus, the firm's profits are equal to $p_{0} \pi_{L}\left(q_{L}^{*}\right)$.

Alternatively, if $p_{0}<p^{C}$, then the unique optimal menu is

$$
\left\{\left(x_{H}^{C}, q_{H}^{C}\right),\left(x_{L}^{C}, q_{L}^{C}\right)\right\}=\left\{\left(\theta_{H} q_{H}^{C}+\alpha, q_{H}^{C}\right),\left(\theta_{L} q_{L}^{*}+\Delta \theta q_{H}^{C}+\alpha, q_{L}^{*}\right)\right\}
$$

for some $q_{H}^{C} \in\left(0, q_{H}^{*}\right)$. The high-cost worker accepts the first contract and obtains a payoff equal to 0 . The low-cost worker is indifferent between the two contracts (therefore, he obtains a payoff equal to $\Delta \theta q_{H}^{C}$ ) and accepts the second contract. In this case, the firm's commitment profits are equal to

$$
p_{0}\left[v\left(q_{L}^{*}\right)-\theta_{L} q_{L}^{*}-\Delta \theta q_{H}^{C}-\alpha\right]+\left(1-p_{0}\right)\left[v\left(q_{H}^{C}\right)-\theta_{H} q_{H}^{C}-\alpha\right] .
$$

Finally, if $p_{0}=p^{C}$, then there are two optimal deterministic mechanisms: $\left\{\left(\theta_{L} q_{L}^{*}+\right.\right.$ $\left.\left.\alpha, q_{L}^{*}\right)\right\}$ and $\left\{\left(x_{H}^{C}, q_{H}^{C}\right),\left(x_{L}^{C}, q_{L}^{C}\right)\right\}$ as in (1). In addition, there is a continuum of optimal

\footnotetext{
${ }^{7}$ To see why $p^{C}>\hat{p}$, let $V_{F}^{C}(p)$ be the commitment payoff of the firm when the prior is $p$. The function $V_{F}^{C}(\cdot)$ is strictly increasing. If $p^{C} \leq \hat{p}$, then we obtain the contradiction $V_{F}^{C}\left(p^{C}\right)=p^{C} \pi_{L}\left(q_{L}^{*}\right) \leq \hat{p} \pi_{L}\left(q_{L}^{*}\right)=$ $\pi_{H}\left(q_{H}^{*}\right)=V_{F}^{C}(0)$.
} 
random mechanisms, since any randomization between the two optimal deterministic mechanisms is also an optimal mechanism.

Suppose that $p_{0} \leq p^{C}$. In the dynamic game with limited commitment, it is impossible to implement the optimal mechanism of the form $\left\{\left(x_{H}^{C}, q_{H}^{C}\right),\left(x_{L}^{C}, q_{L}^{C}\right)\right\}$ in every period. This is because, as shown in Lemma 1, the firm's continuation payoff must be equal to $\pi_{i}\left(q_{i}^{*}\right)$ as soon as the firm discovers that the worker is of type $i .^{8}$

Finally, the firm's payoff in any PBE must be equal to $p_{0} \pi_{L}\left(q_{L}^{*}\right)$ whenever $p_{0} \geq p^{C}$. It cannot be smaller, because of Lemma 1 , and it cannot be larger, because $\left\{\left(\theta_{L} q_{L}^{*}+\alpha, q_{L}^{*}\right)\right\}$ is an optimal mechanism with commitment. Therefore, if $p_{0} \geq p^{C}$, all PBE share the following feature: the high type quits the relationship in the first period, while the low type accepts the contract $\left(\theta_{L} q_{L}^{*}+\alpha, q_{L}^{*}\right)$ in every period.

\section{EXISTENCE AND LEARNING}

In this section, we show the existence of PBE for generic values of the parameters. We also identify the conditions under which the firm is able to learn the worker's type (with and without firing). In particular, if the parties are impatient, then learning is possible for any prior. In contrast, if the parties are sufficiently patient, then learning takes place only when the firm is willing to fire the high-cost worker.

We start with a general result that holds in every PBE: the low-cost worker's relationship with the firm lasts forever.

Lemma 2. Fix a PBE $(\sigma, \mu)$ and an arbitrary history $h^{t}$. For every menu $m_{t}$ offered by the firm at $h^{t}$, we have

$$
\sum_{\left(x_{t}, q_{t}\right) \in m_{t}} \sigma_{t}^{L}\left(\left(x_{t}, q_{t}\right) \mid h^{t}, m_{t}\right)=1 .
$$

We omit the proof of this simple result and provide only an intuitive discussion. Suppose, by contradiction, that there are a $\operatorname{PBE}(\sigma, \mu)$ and a history $h^{t}$ at which the low type rejects all the contracts in the firm's menu with a positive probability. This implies that the interaction with the high type must yield a strictly positive continuation payoff to the firm; otherwise its continuation payoff would be strictly smaller than $\mu\left(h^{t}\right) \pi_{L}\left(q_{L}^{*}\right)$, contradicting Lemma 1. Clearly, a strictly positive continuation payoff is possible only if the high type is expected to deliver a strictly positive (discounted) quality in the future. This and the individual rationality of the high type's behavior imply that the low type's decision to quit is not optimal, as he can guarantee a strictly positive payoff by imitating the high-cost worker at $h^{t}$ and in every future period.

Suppose that the firm is interested in separating the two types and learning the worker's cost. This requires the existence of two decisions, one of which is taken only by the high type, while the other is taken only by the low type. After observing the first (second) decision, the firm becomes convinced that the worker's cost is high (low).

In light of Lemma 2, there are two ways in which separation can take place in equilibrium. One possibility is separation with firing: the high type quits the relationship and

\footnotetext{
${ }^{8}$ Notice that $\pi_{H}\left(q_{H}^{C}\right)<\pi_{H}\left(q_{H}^{*}\right)$ since $q_{H}^{C}<q_{H}^{*}$. Also $v\left(q_{L}^{*}\right)-\theta_{L} q_{L}^{*}-\alpha-\Delta \theta q_{H}^{C}<\pi_{L}\left(q_{L}^{*}\right)$ since $q_{H}^{C}>0$.
} 
one of the contracts in the firm's menu is only accepted by the low type. The other possibility is separation with employment: one contract in the firm's menu is only accepted by the high type, while another contract is only accepted by the low type.

Our next result shows that separation with employment cannot occur for values of the discount factor greater than the critical threshold $\hat{\delta}:=\frac{1}{1+q_{H}^{*}}$.

Lemma 3. Suppose that $\delta>\hat{\delta}$ and let $(\sigma, \mu)$ be an arbitrary PBE of the game. It is impossible to find a history $\left(h^{t}, m_{t}\right)$ (on- or off-path) that satisfies the following properties:

(i) We have $\mu\left(h^{t}\right) \in(0,1)$.

(ii) There exists a contract $\left(x_{H}, q_{H}\right)$ in $m_{t}$ for which $\sigma_{t}^{H}\left(\left(x_{H}, q_{H}\right) \mid h^{t}, m_{t}\right)>0$ and $\sigma_{t}^{L}\left(\left(x_{H}, q_{H}\right) \mid h^{t}, m_{t}\right)=0$.

(iii) There exists a contract $\left(x_{L}, q_{L}\right)$ in $m_{t}$ for which $\sigma_{t}^{L}\left(\left(x_{L}, q_{L}\right) \mid h^{t}, m_{t}\right)>0$ and $\sigma_{t}^{H}\left(\left(x_{L}, q_{L}\right) \mid h^{t}, m_{t}\right)=0$.

We provide the intuition for this result; see the Supplemental Material, available in a supplementary file on the journal website, http://econtheory.org/supp/2449/ supplement.pdf, for the proof. By contradiction, suppose that at $h^{t}$, the belief is nondegenerate and the firm's menu contains a contract $\left(x_{i}, q_{i}\right)$ that is accepted with positive probability only by the type $i \in\{H, L\}$. Following the acceptance of this contract, the firm's belief assigns probability 1 to type $i .^{9}$ Furthermore, in equilibrium, type $i$ selects the efficient contract $\left(\theta_{i} q_{i}^{*}+\alpha, q_{i}^{*}\right)$ in every period after $t$. If the discount factor is above $\hat{\delta}$, it is impossible to find two contracts, $\left(x_{H}, q_{H}\right)$ and $\left(x_{L}, q_{L}\right)$, to satisfy the two incentive compatibility constraints. In fact, either the low type prefers to imitate the high type (at $h^{t}$ and in every future period) or the high type has an incentive to adopt the "take the money and run" strategy (i.e., the strategy of accepting the generous contract $\left(x_{L}, q_{L}\right)$ and then quitting).

We are now ready to state the main result of this section, which establishes the (generic) existence of PBE.

Proposition 1. For generic values of the parameters, there exists a PBE.

The proof of Proposition 1 (provided in Appendix A) shows how to construct a PBE for all values of $\delta$ outside a set of discount factors that can contain at most two elements (the values of these two elements depend on the primitives $\theta_{H}, \theta_{L}, \alpha$, and $\left.v(\cdot)\right) .{ }^{10}$ For the remainder of the paper, we assume that the discount factor $\delta$ does not belong to this (possibly empty) set.

The equilibrium that we construct satisfies a number of properties. First, the equilibrium is "almost Markovian," in the sense that the parties' behavior in period $t$ depends

\footnotetext{
${ }^{9}$ Notice that in a PBE, the beliefs must satisfy this condition at all histories, including those that are off-path.

${ }^{10}$ Our formal argument does not cover the values of $\delta$ at which the mapping $V^{1}:[0,1] \rightarrow \mathbb{R}$ defined in (S1) (see the Supplemental Material) simultaneously satisfies $V^{1}(0)=\pi_{H}\left(q_{H}^{*}\right)$ and $\partial_{+} V^{1}(0)=0$. We show that there can be at most two such values of $\delta$.
} 
on the firm's belief and their actions in period $t-1$ (the history up to period $t-2$ affects the behavior in period $t$ only through the belief). Second, the high type plays a pure strategy and his equilibrium payoff is equal to 0 . Third, the menu proposed by the firm (at any history) contains at most two contracts. Finally, the firm adopts a deterministic behavior at on-path histories.

We now introduce some definitions to illustrate our equilibrium. First, we say that there is a pooling allocation if the firm offers the menu $\left\{\left(\theta_{H} q_{H}^{*}+\alpha, q_{H}^{*}\right)\right\}$ in every period and both types accept the contract $\left(\theta_{H} q_{H}^{*}+\alpha, q_{H}^{*}\right)$ (with probability 1$)$. We also say that there is a firing allocation if the firm offers the menu $\left\{\left(\theta_{L} q_{L}^{*}+\alpha, q_{L}^{*}\right)\right\}$ in every period, the high type quits in the first period, and the low type accepts the contract $\left(\theta_{L} q_{L}^{*}+\alpha, q_{L}^{*}\right)$ in every period. Finally, we say that there is a sequentially screening allocation if the firm offers a menu with two contracts in every period in which its belief is nondegenerate. Furthermore, the high type accepts the first contract with probability 1, while the low type accepts the second contract with a strictly positive probability (if this probability is less than 1, the low type randomizes between the two contracts). Therefore, in a sequentially screening allocation, either the firm learns that the worker's type is low or it becomes more confident that the worker's type is high.

To illustrate our construction, it is convenient to distinguish between the case $\delta \leq \hat{\delta}$ and the case $\delta>\hat{\delta}$. We start with the first case. We assume (without loss) that the firm offers the menu $\left\{\left(\theta_{H} q_{H}^{*}+\alpha, q_{H}^{*}\right)\right\}$ when the belief $p$ is equal to 0 . Also, the firm offers the menu $\left\{\left(\theta_{L} q_{L}^{*}+\alpha, q_{L}^{*}\right)\right\}$ when $p \geq p^{C}$. For any belief $p \in\left(0, p^{C}\right)$, we first provide three options to the firm: (i) offering a pooling menu, i.e., a menu with one contract that is accepted by both types; (ii) offering a firing menu, i.e., a menu with one contract that induces separation with firing (where the low type accepts the contract while the high type quits); (iii) offering a menu with two contracts to induce separation with employment (i.e., with probability 1 , the two types choose different contracts).

Clearly, the optimal pooling menu is $\left\{\left(\theta_{H} q_{H}^{*}+\alpha, q_{H}^{*}\right)\right\}$, while the optimal firing menu is $\left\{\left(\theta_{L} q_{L}^{*}+\alpha, q_{L}^{*}\right)\right\}$. In case (iii), we choose the two contracts to maximize the firm's payoff subject to the incentive compatibility (IC) and the individual rationality (IR) constraints. Notice that after separation with employment, the firm's belief is either 0 or 1 . In both cases, the firm's behavior is known and we can compute the two types' continuation payoffs. As in the standard mechanism design problem with commitment, the optimal menu in case (iii) is such that both the low type's IC constraint and the high type's IR constraint are binding.

We construct the firm's value function $V(\cdot ; 1)$ and the low type's payoff correspondence $\Phi(\cdot ; 1)$ when the firm is forced to choose one of the three options above. ${ }^{11} \mathrm{We}$ take $V(\cdot ; 1)$ and $\Phi(\cdot ; 1)$ as given and offer the firm the possibility of probabilistic separation with employment. This means that the firm offers two contracts. The high type accepts the first contract with probability 1, while the low type randomizes between the contracts. After this round of probabilistic separation, the firm is again forced to use the three options above and, therefore, the parties' continuation payoffs are given by

\footnotetext{
${ }^{11}$ For some values of the beliefs $p$, the solution to the firm's problem is not unique and different solutions generally yield different payoffs to the low type (hence, we use the correspondence $\Phi(\cdot ; 1)$ ).
} 
$V(\cdot ; 1)$ and $\Phi(\cdot ; 1)$. In the probabilistic separation phase, we select the two contracts and the low type's behavior (i.e., the probability of accepting each contract) to maximize the firm's payoff subject, of course, to the IC and IR constraints. As usual, the solution to the optimization problem satisfies the low type's IC constraint with equality and, therefore, randomizing between the two contracts is indeed optimal for the low-cost worker.

The possibility of probabilistic separation defines a new value function $V(\cdot ; 2)$ and a new payoff correspondence $\Phi(\cdot ; 2)$. If $V(p ; 2)=V(p ; 1)$ for every $p \in[0,1]$, then we stop the process, as the firm does not benefit from probabilistic separation. Alternatively, if $V(p ; 2)>V(p ; 1)$ (it is possible to construct examples for which this is the case), then we allow for an additional round of probabilistic separation with employment.

We continue the process (allowing, at each iteration, for a new round of probabilistic separation) until we find a fixed point $(V(\cdot), \Phi(\cdot))$. We show that for generic values of the parameters, a fixed point exists and is achieved after finitely many iterations. Moreover, our proof shows that if we fix the parameters $\left(\theta_{H}, \theta_{L}, \alpha, v(\cdot)\right)$, then there is $T$ such that for generic discount factors smaller than $1-q_{H}^{*} / q_{L}^{*}$, the number of iterations is smaller than $T$ (see Corollary 1 below for the implications of this result).

The pair $(V(\cdot), \Phi(\cdot))$ allows us to construct a simple equilibrium. For each belief $p$, the parties behave according to the solution of the firm's optimization problem (which yields the payoff $V(p)$ to the firm). The solution consists of the optimal menu and the decision of the worker. In particular, if the optimal menu is $\left\{\left(\theta_{H} q_{H}^{*}+\alpha, q_{H}^{*}\right)\right\}$, then both types accept the contract. If the optimal menu is $\left\{\left(\theta_{L} q_{L}^{*}+\alpha, q_{L}^{*}\right)\right\}$, then only the low type accepts the contract. Finally, if the optimal menu contains two contracts, then the high type accepts the first contract (with probability 1 ) while the low type accepts the second contract with probability in $(0,1]$ (this probability is part of the solution to the optimization problem).

The proof of Proposition 1 also specifies the parties' off-path behavior and shows that unilateral deviations are not profitable.

We conclude the discussion of the case of a low discount factor by pointing out a property of our equilibria. Fix a PBE $(\sigma, \mu)$. We say that there is full learning by period $t$ if for any $t^{\prime} \geq t$ and for any on-path public history $h^{t^{\prime}}$, the belief $\mu\left(h^{t^{\prime}}\right)$ is either 0 or 1 . This means that all the uncertainty about the worker's ability is resolved by period $t$.

Our construction shows that when the parties are not too patient, the firm never chooses the pooling allocation. Depending on the value of the prior, the firm prefers either the sequentially screening allocation (if the prior is low) or the firing allocation (if the prior is high). In both cases, there is full learning. Moreover, our proof shows that the number of periods until the worker completely reveals his private information is uniformly bounded. ${ }^{12}$ Formally, we obtain the following result.

Corollary 1. Fix the parameters $\left(\theta_{H}, \theta_{L}, \alpha, v(\cdot)\right)$. There exists $T \in\{1,2, \ldots\}$ such that for any prior $p_{0}$ and for generic values of the discount factor smaller than $1-q_{H}^{*} / q_{L}^{*}$, there exists a PBE with full learning by period $T$.

\footnotetext{
${ }^{12}$ This follows from the argument provided at the end of the proof of Lemma 8.
} 
Finally, as $\delta$ shrinks to 0 , the firm's equilibrium payoff converges to the payoff of the optimal mechanism with commitment. This is a general property that holds for all PBE. ${ }^{13}$

We now turn to the case the case $\delta>\hat{\delta}$. Recall that in this case, separation with employment is not feasible. Therefore, the firm is unable to implement a sequentially screening allocation. As a result, the firm chooses between the pooling and the firing allocation. The equilibrium that we construct takes a very simple form. If the prior is weakly higher than $\hat{p}$, the firm offers the optimal firing menu $\left\{\left(\theta_{L} q_{L}^{*}+\alpha, q_{L}^{*}\right)\right\}$ in every period and the high-cost worker quits in the first period. Thus, the equilibrium is with full learning by period one. In contrast, if the prior is lower than $\hat{p}$, the firm offers the optimal pooling menu $\left\{\left(\theta_{H} q_{H}^{*}+\alpha, q_{H}^{*}\right)\right\}$ in every period and never updates (along the equilibrium path) its belief.

The analysis in this section shows that when the parties are sufficiently patient, the ratchet effect has a strong impact on equilibrium behavior. In particular, it suggests that, for sufficiently large values of $\delta$, the firm can learn the worker's cost only by firing the high type. As we see in the next section, this is not a special feature of our equilibrium, but a much more general result.

\section{LIMIT UNIQUENESS}

In the last section, we constructed a simple equilibrium for the case with sufficiently patient parties $(\delta>\hat{\delta})$. This equilibrium implements the pooling allocation when the prior is lower than $\hat{p}$ and the firing allocation when the prior is higher than $\hat{p}$. Our construction relies on the fact that a sequentially screening allocation is not feasible when the discount factor is above $\hat{\delta}$. However, the firm could, in principle, employ more complex dynamic screening strategies. For instance, one could imagine an equilibrium in which two or more contracts in the firm's menu are accepted with positive, but different, probabilities by the two types. In this case, the firm's belief could increase without jumping to 1 , as occurs in a sequentially screening allocation. This raises the question of whether there are other equilibrium outcomes, in addition to the one identified in Section 4. Moreover, can the firm do better than just offering the optimal pooling menu or the optimal firing menu?

We show that in the limit, as the parties become arbitrarily patient, there exists a unique equilibrium outcome. This outcome coincides with the equilibrium outcome described in Section 4 (for the case $\delta>\hat{\delta}$ ). First, if the prior is above $\hat{p}$, the limiting equilibrium allocation is firing and the high type quits the relationship without delay. ${ }^{14}$ In contrast, if the prior is below $\hat{p}$, the limiting equilibrium allocation is pooling and there is no learning. ${ }^{15}$

\footnotetext{
${ }^{13}$ For brevity, we omit the proof of this simple finding.

${ }^{14}$ Recall that with commitment, the high type is fired if the prior is above $p^{C}$ and that $p^{C}$ is larger than $\hat{p}$. Therefore, limited commitment enlarges (in the limit, as $\delta$ goes to 1 ) the region of beliefs for which the equilibrium allocation is firing.

${ }^{15}$ When the prior is equal to $\hat{p}$, the limiting equilibrium outcome is not uniquely pinned down, as there are equilibria implementing the pooling allocation, the firing allocation, and convex combinations of such allocations.
} 


\subsection{High belief case: $p>\hat{p}$}

We first study the limiting equilibrium outcome when the prior is above $\hat{p}$. To do so, we define the notion of firing region. Recall that $\mathbb{T}$ denotes the random time at which the worker quits the relationship.

Definition 2. The interval [ $p, 1]$ is a firing region if there exist $\bar{K}>0$ and $\bar{\delta}<1$ such that the following statement holds. Fix $\delta>\bar{\delta}$, an arbitrary $\operatorname{PBE}(\sigma, \mu)$, and a history $h^{t}$ at which $\mu\left(h^{t}\right) \geq p$. Then we have

(i) $\mathbb{E}_{(\sigma, \mu)}\left[(1-\delta) \sum_{\tau=t}^{\mathbb{T}-1} \delta^{\tau-t} \mid h^{t}, H\right]<\bar{K}(1-\delta)$

(ii) $V_{F}\left(h^{t} ;(\sigma, \mu), H\right)<\bar{K}(1-\delta)$

(iii) $W_{L}\left(h^{t} ;(\sigma, \mu)\right)<\bar{K}(1-\delta)$.

When the firm's belief falls in a firing region, the expected discounted length of the firm's relationship with the high type vanishes as the parties become arbitrarily patient (property (i)). Furthermore, both the firm's payoff from the interaction with the high type and the low type's payoff shrink to 0 as $\delta$ goes to 1 (properties (ii) and (iii), respectively). Although not part of its definition, a firing region satisfies the additional property that the equilibrium allocation must converge to the firing allocation. ${ }^{16}$

We are now ready to state the main result of this section.

Proposition 2. For every $p>\hat{p}$, the interval $[p, 1]$ is a firing region.

The proof of Proposition 2 is by induction. The interval $\left[p^{C}, 1\right]$ is a firing region since, for any $\delta$, the unique equilibrium allocation is firing. This is the first step of the proof. In the inductive step, we take an arbitrary firing region $[p, 1]$ and extend it to include beliefs lower than $p$.

The following preliminary result plays a key role in the proof of the inductive step.

Lemma 4. Suppose that $[p, 1]$ is a firing region. There exist $K>0$ and $\tilde{\delta}<1$ such that for every $\delta>\tilde{\delta}$, for every $P B E(\sigma, \mu)$, and for every history $h^{t}$, with $\mu\left(h^{t}\right)<p$, the following statement holds. Suppose that at $h^{t}$, the firm offers a menu $m_{t}$ containing a contract $\left(x_{t}^{L}, q_{t}^{L}\right)$ accepted with positive probability and for which

$$
\mu\left(h^{t}, m_{t},\left(x_{t}^{L}, q_{t}^{L}\right)\right) \geq p .
$$

Then we have

(i) $\mathbb{E}_{(\sigma, \mu)}\left[(1-\delta) \sum_{\tau=t}^{\mathbb{T}-1} \delta^{\tau-t} \mid h^{t}, m_{t}, H\right]<K(1-\delta)$

(ii) $V_{F}\left(h^{t}, m_{t} ;(\sigma, \mu), H\right)<K(1-\delta)$

(iii) $W_{L}\left(h^{t}, m_{t} ;(\sigma, \mu)\right)<K(1-\delta)$.

\footnotetext{
${ }^{16}$ Formally, we can take $\bar{K}$ such that for every $\delta>\bar{\delta}$, every $\operatorname{PBE}(\sigma, \mu)$, and every history $h^{t}$ at which $\mu\left(h^{t}\right) \geq p$, we also have $\mathbb{E}_{(\sigma, \mu)}\left[(1-\delta) \sum_{t=0}^{\mathbb{T}-1} \delta^{t}\left|q_{t}-q_{L}^{*}\right| \mid h^{t}, L\right]<\bar{K}(1-\delta)$, and $\mathbb{E}_{(\sigma, \mu)}\left[(1-\delta) \sum_{t=0}^{\mathbb{T}-1} \delta^{t}\left(x_{t}-\right.\right.$ $\left.\left.\theta_{L} q_{L}^{*}-\alpha\right) \mid h^{t}, L\right]<\bar{K}(1-\delta)$.
} 
Consider a history $h^{t}$ at which the firm offers a menu $m_{t}$ containing a contract that leads to a firing region. Lemma 4 provides bounds for the length of the high type's relationship and for the continuation payoffs conditional on the menu $m_{t}$ being offered.

The proof of Lemma 4 is given in Appendix B. The driving force behind Lemma 4 is similar to that behind Lemma 3, which establishes that separation with employment cannot occur for large values of $\delta$. The intuition is as follows. Following the acceptance of a contract $\left(x_{t}^{L}, q_{t}^{L}\right)$ that leads to the firing region, the low type's continuation payoff is close to 0 . Suppose the firm's relationship with the high type is long lasting. In this case, only a large transfer $x_{t}^{L}$ can prevent the low type from mimicking the high type. But then it becomes profitable for the high type to accept the contract $\left(x_{t}^{L}, q_{t}^{L}\right)$ and then quit.

We now turn to the inductive step. For every $p \geq \hat{p}$, let $f(p) \in[0, p-\hat{p}]$ be defined by

$$
\frac{f(p)}{p} \pi_{H}^{\prime}(0)+\left(1-\frac{f(p)}{p}\right) \pi_{H}\left(q_{H}^{*}\right)=(p-f(p)) \pi_{L}\left(q_{L}^{*}\right) .
$$

The function $f:[\hat{p}, 1] \rightarrow[0,1-\hat{p}]$ is strictly increasing and satisfies $f(\hat{p})=0$ and $f(p)<p-\hat{p}$ for every $p>\hat{p} .^{17}$

Lemma 5. Suppose that the interval $[p, 1], p \in(\hat{p}, 1)$, is a firing region. Then $\left[p-\frac{f(p)}{2}, 1\right]$ is also a firing region.

The rest of the section provides the proof of Lemma 5, which consists of several steps. We outline in detail each step and relegate some technical arguments to Appendix B.

The continuation play starting at some history $h^{t}$ is an equilibrium of the original game (when the prior is equal to the firm's belief at $h^{t}$ ). It is, therefore, without loss of generality (and convenient in terms of notation) to establish the three properties of a firing region for the initial history $h^{0}$.

We first show that the expected discounted length of the high type's relationship shrinks to 0 (property (i) of Definition 2). This is the main part of the proof of Lemma 5 because, as we show later, the remaining two properties follow from the first.

Any equilibrium $(\sigma, \mu)$ must satisfy the following two conditions. First, the firm's equilibrium payoff $V_{F}\left(h^{0}\right)$ must be at least equal to $p_{0} \pi_{L}\left(q_{L}^{*}\right)$. Second, the low type must prefer his strategy to mimicking the high type. Thus, $W_{L}\left(h^{0}\right) \geq W_{L H}\left(h^{0}\right)$, where $W_{L H}\left(h^{0}\right)$ denotes the low type's continuation payoff at $h^{0}$ if he mimics the high type (at every history). We show that if $[p, 1]$ is a firing region, the prior $p_{0}$ is above $p-\frac{f(p)}{2}$, and $\delta$ is close to 1 , the two conditions just mentioned can be simultaneously satisfied only if the expected discounted length of the high type's relationship is close to 0 .

We proceed in four steps. The payoffs $V_{F}\left(h^{0}\right), W_{L}\left(h^{0}\right)$, and $W_{L H}\left(h^{0}\right)$, and the expected length of the relationship are complicated objects since they depend on the entire history of the game. Instead of working directly with these variables, we replace them with some bounds that are easy to express and compare. We do this in the first three steps. In Step 1, we show that it is without loss of generality to restrict attention to a class of simple equilibria. In Step 2, we provide bounds to the continuation payoffs

\footnotetext{
${ }^{17}$ Recall that the function $\pi_{H}(\cdot)$ is concave and, therefore, $\pi_{H}^{\prime}(0)>\pi_{H}^{\prime}(0) q_{H}^{*} \geq \pi_{H}\left(q_{H}^{*}\right)$.
} 
and to the length of the relationship with the high type. These bounds are derived by changing the timing of the transfers in the equilibrium of the game. In Step 3, we introduce an auxiliary game that allows for a simpler expression of these bounds. Finally, in Step 4, we use these bounds to establish property (i) of Definition 2.

Step 1: Restriction to a class of simple equilibria. We now show that to establish property (i) of Definition 2 it is without loss of generality to restrict attention to equilibria in which (a) the firm's strategy in the first period is pure (i.e., the firm does not randomize among different menus at $t=0$ ) and (b) the high type's equilibrium payoff is equal to zero. To see why restriction (a) is without loss, suppose that $\left(\left(\sigma^{F}, \sigma^{H}, \sigma^{L}\right), \mu\right)$ is a PBE and $m_{0}$ is a menu offered with positive probability by the firm at $t=0\left(\sigma_{0}^{F}\left(m_{0} \mid h_{0}\right)>\right.$ 0 ). Let $\tilde{\sigma}^{F}$ be the strategy that is identical to $\sigma^{F}$ in every period except the first, in which the firm instead offers the menu $m_{0}$ with certainty $\left(\tilde{\sigma}_{0}^{F}\left(m_{0} \mid h_{0}\right)=1\right)$. The assessment $\left(\left(\tilde{\sigma}^{F}, \sigma^{H}, \sigma^{L}\right), \mu\right)$ is also a PBE. Furthermore, the outcome of the equilibrium $\left(\left(\tilde{\sigma}^{F}, \sigma^{H}, \sigma^{L}\right), \mu\right)$ coincides with the continuation outcome of $\left(\left(\sigma^{F}, \sigma^{H}, \sigma^{L}\right), \mu\right)$ after the firm proposes the contract $m_{0}$. Therefore, if it is impossible to establish the first property for arbitrary $\mathrm{PBE}$, then it is also impossible to establish the property for the class of equilibria that satisfy restriction (a).

We show next that restriction (b) is also without loss of generality. Suppose that $(\sigma, \mu)$ is a PBE in which the firm offers the menu $m_{0}$ (with probability 1 ) in the first period and that yields a strictly positive payoff $W_{H}\left(h^{0} ;(\sigma, \mu)\right)$ to the high type. Then it is possible to construct a new $\operatorname{PBE}(\tilde{\sigma}, \tilde{\mu})$ that is outcome equivalent to $(\sigma, \mu)$, except for the fact that the first-period transfers are uniformly decreased by $(1-\delta)^{-1} W_{H}\left(h^{0} ;(\sigma, \mu)\right)$. In other words, in the first period, the firm replaces every contract $\left(x_{0}, q_{0}\right)$ in the menu $m_{0}$ with the contract $\left(x_{0}-(1-\delta)^{-1} W_{H}\left(h^{0} ;(\sigma, \mu)\right), q_{0}\right){ }^{18}$ Finally, notice that the first property of a firing region does not depend on equilibrium transfers.

Step 2: Change in the timing of transfers. This step provides bounds to the payoffs and to the length of the relationship that depend only on the quality of the goods delivered by the worker. Let $\tilde{\mathbb{T}} \in \mathbf{N} \cup\{\infty\}$ denote the random time that stops the play at the first history $\left(h^{\tilde{\mathbb{T}}}, m_{\tilde{\mathbb{T}}}\right)$ at which the menu $m_{\tilde{\mathbb{T}}}$ contains a contract $\left(x_{\tilde{\mathbb{T}}}, q_{\tilde{\mathbb{T}}}\right)$ accepted with positive probability and for which $\mu\left(h^{\tilde{\mathbb{T}}}, m_{\tilde{\mathbb{T}}},\left(x_{\tilde{\mathbb{T}}}, q_{\tilde{\mathbb{T}}}\right)\right) \geq p$ (we set $\tilde{\mathbb{T}}=\infty$ if the event does not occur in finite time). We claim that there exist $K>0$ and $\bar{\delta}<1$ such that for $\delta>\bar{\delta}$, we have

$$
\begin{aligned}
& V_{F}\left(h^{0}\right) \leq \bar{V}_{F}\left(h^{0}\right):=\mathbb{E}_{(\sigma, \mu)}\left[(1-\delta) \sum_{t=0}^{\tilde{\mathbb{T}}-1} \delta^{t} \pi_{H}\left(q_{t}\right)+\mathbb{I}_{\{\tilde{\mathbb{T}}<\infty\}} \delta^{\tilde{\mathbb{T}}} \mu\left(h^{\tilde{\mathbb{T}}}\right) \pi_{L}\left(q_{L}^{*}\right)\right]+K(1-\delta) \\
& W_{L}\left(h^{0}\right) \leq \bar{W}_{L}\left(h^{0}\right):=\mathbb{E}_{(\sigma, \mu)}\left[(1-\delta) \sum_{t=0}^{\tilde{\mathbb{T}}-1} \delta^{t} \Delta \theta q_{t} \mid L\right]+K(1-\delta) \\
& W_{L H}\left(h^{0}\right) \geq \underline{W}_{L H}\left(h^{0}\right):=\mathbb{E}_{(\sigma, \mu)}\left[(1-\delta) \sum_{t=0}^{\tilde{\mathbb{T}}-1} \delta^{t} \Delta \theta q_{t} \mid H\right]-K(1-\delta)
\end{aligned}
$$

\footnotetext{
${ }^{18}$ In the new equilibrium $(\tilde{\sigma}, \tilde{\mu})$, each type of worker accepts the contract $\left(x_{0}-(1-\right.$ $\left.\delta)^{-1} W_{H}\left(h^{0} ;(\sigma, \mu)\right), q_{0}\right)$ with the same probability with which he accepts the contract $\left(x_{0}, q_{0}\right)$ in the original equilibrium $(\sigma, \mu)$.
} 
and

$$
\mathbb{E}_{(\sigma, \mu)}\left[(1-\delta) \sum_{t=0}^{\mathbb{T}-1} \delta^{t} \mid h^{0}, H\right] \leq \mathbb{E}_{(\sigma, \mu)}\left[(1-\delta) \sum_{t=0}^{\tilde{\mathbb{T}}-1} \delta^{t} \mid h^{0}, H\right]+K(1-\delta) .
$$

The bounds on the payoffs provided in (3) are derived as follows (the formal derivation is provided in the Appendix). Recall that the high type's equilibrium payoff $W_{H}\left(h^{0}\right)$ is equal to 0 . Let $m_{0}$ be the menu offered by the firm at $h^{0}$ and consider any contract $\left(x_{0}, q_{0}\right) \in m_{0}$ accepted by the high type with positive probability. Let $h^{1}$ denote the history $\left(h^{0}, m_{0},\left(x_{0}, q_{0}\right)\right)$. If $W_{H}\left(h^{1}\right)=0$, then we have $x_{0}=\theta_{H} q_{0}+\alpha$. If instead $W_{H}\left(h^{1}\right)>0$, then we increase the transfer $x_{0}$ by the amount $\frac{\delta}{(1-\delta)} W_{H}\left(h^{1}\right)$. The new transfer is equal to $\theta_{H} q_{0}+\alpha$. At the same time, for every menu $m_{1}$ offered at $h^{1}$, we decrease all the transfers of the contracts in $m_{1}$ by the amount $\frac{1}{(1-\delta)} W_{H}\left(h^{1}, m_{1}\right)$. These changes leave all parties' continuation payoffs unchanged. We repeat this procedure in periods $1, \ldots, \tilde{\mathbb{T}}-1$. Thus, for every $t=0, \ldots, \tilde{\mathbb{T}}-1$, the new transfer in period $t$ is equal to $\theta_{H} q_{t}+\alpha$. Furthermore, it follows from Lemma 4 that at the history $\left(h^{\tilde{\mathbb{T}}}, m_{\tilde{\mathbb{T}}}\right)$, if $\delta$ is close to 1 , the firm's continuation payoff is close to $\mu\left(h^{\tilde{\mathbb{T}}}\right) \pi_{L} q_{L}^{*}$, while the worker's payoff is close to $0 .{ }^{19}$ Combining the expressions of the new transfers with the findings on the continuation payoffs we obtain the bounds in (3).

The bound on the expected length of the high type's relationship (inequality (4)) follows directly from Lemma 4 (property (i)).

Step 3: The auxiliary game. We introduce an auxiliary game (a direct mechanism) that replicates the equilibrium outcome from period 0 through period $\tilde{\mathbb{T}}-1$ and implements the payoffs $\bar{V}_{F}\left(h^{0}\right), \bar{W}_{L}\left(h^{0}\right)$, and $\underline{W}_{L H}\left(h^{0}\right)$. In particular, we consider a mechanism in which the worker reveals his type to a designer. The designer selects a history and one of two messages, $\mathfrak{m}_{0}$ and $\mathfrak{m}_{p}$ (see below for more details about the mechanism). Let $\Upsilon_{z}$, for $z \in\{0, p\}$, denote the expected discounted length of the relationship conditional on the message $\mathfrak{m}_{z}$, and let $\tilde{q}_{z}$ denote the expected discounted quality of the good conditional on $\mathfrak{m}_{z}$. Using the auxiliary game, we show that

$$
\begin{aligned}
\bar{V}_{F}\left(h^{0}\right) & \leq \breve{V}_{F}\left(h^{0}\right) \\
& :=\left(1-\frac{p_{0}}{p}\right) \Upsilon_{0} \pi_{H}\left(\tilde{q}_{0}\right)+\frac{p_{0}}{p}\left[\Upsilon_{p} \pi_{H}\left(\tilde{q}_{p}\right)+\left(1-\Upsilon_{p}\right) p \pi_{L}\left(q_{L}^{*}\right)\right]+K(1-\delta) \\
\bar{W}_{L}\left(h^{0}\right) & =\Upsilon_{p} \tilde{q}_{p} \Delta \theta+K(1-\delta) \\
\underline{W}_{L H}\left(h^{0}\right) & =\left(\frac{1}{1-p_{0}}\right)\left(1-\frac{p_{0}}{p}\right) \Upsilon_{0} \tilde{q}_{0} \Delta \theta+\left(\frac{1-p}{1-p_{0}}\right)\left(\frac{p_{0}}{p}\right) \Upsilon_{p} \tilde{q}_{p} \Delta \theta-K(1-\delta)
\end{aligned}
$$

and

$$
\begin{aligned}
& \mathbb{E}_{(\sigma, \mu)}\left[(1-\delta) \sum_{t=0}^{\mathbb{T}-1} \delta^{t} \mid h^{0}, H\right] \\
& \quad \leq\left(\frac{1}{1-p_{0}}\right)\left(1-\frac{p_{0}}{p}\right) \Upsilon_{0}+\left(\frac{1-p}{1-p_{0}}\right)\left(\frac{p_{0}}{p}\right) \Upsilon_{p}+K(1-\delta) .
\end{aligned}
$$

\footnotetext{
${ }^{19}$ In fact, the high type's payoff is bounded above by the low type's payoff, which, in turn, is close to 0 (see property (iii) in Lemma 4).
} 
This greatly simplifies our problem since it reduces to four the number of endogenous variables $\left(\Upsilon_{z}\right.$ and $\left.\tilde{q}_{z}, z \in\{0, p\}\right)$ in the expressions of the bounds. We then use the expressions in (5) and (6) to show that the inequalities $\breve{V}_{F}\left(h^{0}\right) \geq p_{0} \pi_{L}\left(q_{L}^{*}\right)$ (recall that $\left.\breve{V}_{F}\left(h^{0}\right) \geq \bar{V}_{F}\left(h^{0}\right) \geq V_{F}\left(h^{0}\right)\right)$ and $\bar{W}_{L}\left(h^{0}\right) \geq \underline{W}_{L H}\left(h^{0}\right)$ can be satisfied only if the right hand side of inequality (6) shrinks to 0 (as $\delta$ goes to 1 ), establishing, therefore, the first property of a firing region.

We now describe the direct mechanism. The worker reveals his private information to a designer who, in turn, chooses an outcome and reports it to the firm. The outcome consists of a history $h^{\tilde{\mathbb{T}}}$ of the game and a message in $\left\{\mathfrak{m}_{0}, \mathfrak{m}_{p}\right\}$. The designer chooses the outcomes in such a way that upon receiving message $\mathfrak{m}_{z}$, the firm's belief is equal to $z$. Moreover, the likelihoods of the histories reflect those from the equilibrium of the game. Specifically, if the worker announces the low type, then the designer chooses the outcome $\left(h^{\tilde{\mathbb{T}}}, \mathfrak{m}_{p}\right)$ with probability $\operatorname{Pr}\left(h^{\tilde{\mathbb{T}}} \mid L\right)$, the probability of the history $h^{\tilde{\mathbb{T}}}$ when the worker's type is low and the parties play the equilibrium $(\sigma, \mu)$. Alternatively, if the worker announces the high type, then the designer chooses the outcome $\left(h^{\tilde{\mathbb{T}}}, \mathfrak{m}_{p}\right)$ with probability $\operatorname{Pr}\left(h^{\tilde{\mathbb{T}}} \mid H\right) \frac{\mu\left(h^{\tilde{\mathbb{T}}}\right)(1-p)}{\left(1-\mu\left(h^{\tilde{\mathbb{T}}}\right)\right) p}$ and the outcome $\left(h^{\tilde{\mathbb{T}}}, \mathfrak{m}_{0}\right)$ with probability $\operatorname{Pr}\left(h^{\tilde{\mathbb{T}}} \mid H\right)[1-$ $\left.\frac{\mu\left(h^{\tilde{\mathbb{T}}}\right)(1-p)}{\left(1-\mu\left(h^{\tilde{\mathbb{T}}}\right)\right) p}\right]$.

We now turn to the payoffs of the firm and the low type. The firm's payoff depends only on the outcome and not on the worker's report. Consider an arbitrary history $h^{\tilde{\mathbb{T}}}=$ $\left(m_{0},\left(x_{0}, q_{0}\right), \ldots, m_{\tilde{\mathbb{T}}},\left(x_{\tilde{\mathbb{T}}-1}, q_{\tilde{\mathbb{T}}-1}\right)\right)$. If the outcome is $\left(h^{\tilde{\mathbb{T}}}, \mathfrak{m}_{p}\right)$, the firm's payoff is equal to

$$
(1-\delta) \sum_{t=0}^{\tilde{\mathbb{T}}-1} \delta^{t} \pi_{H}\left(q_{t}\right)+\mathbb{I}_{\{\tilde{\mathbb{T}}<\infty\}} \delta^{\tilde{\mathbb{T}}} p \pi_{L}\left(q_{L}^{*}\right)+K(1-\delta) .
$$

If the outcome is $\left(h^{\tilde{\mathbb{T}}}, \mathfrak{m}_{0}\right)$, the firm's payoff is equal to

$$
(1-\delta) \sum_{t=0}^{\tilde{\mathbb{T}}-1} \delta^{t} \pi_{H}\left(q_{t}\right)+K(1-\delta)
$$

We conclude that if every type reveals his type truthfully, then the firm's expected payoff is equal to $\bar{V}_{F}\left(h^{0}\right)$.

Consider now the low type. His payoff depends both on the outcome and on his report. First, if the outcome is either $\left(h^{\tilde{\mathbb{T}}}, \mathfrak{m}_{p}\right)$ or $\left(h^{\tilde{\mathbb{T}}}, \mathfrak{m}_{0}\right)$, with $h^{\tilde{\mathbb{T}}}=\left(m_{0},\left(x_{0}, q_{0}\right), \ldots, m_{\tilde{\mathbb{T}}}\right.$, $\left.\left(x_{\tilde{\mathbb{T}}-1}, q_{\tilde{\mathbb{T}}-1}\right)\right)$, then the low type obtains a payoff equal to

$$
(1-\delta) \sum_{t=0}^{\tilde{\mathbb{T}}-1} \delta^{t} \Delta \theta q_{t}
$$

In addition, the low type obtains an extra payoff equal to $K(1-\delta)$ if he is honest, and equal to $-K(1-\delta)$ if he lies to the designer. It follows that the low type's expected payoff is equal to $\bar{W}_{L}\left(h^{0}\right)$ if he reveals his type truthfully and is equal to $\underline{W}_{L H}\left(h^{0}\right)$ if he lies to the designer. Finally, we assume that the high type is committed to truthfully reporting his type to the designer. 
It is natural to ask why we introduced the messages $\mathfrak{m}_{0}$ and $\mathfrak{m}_{p}$ in the mechanism, given that they do not affect the worker's payoffs and the firm is a passive player in the auxiliary game. The reason for this is that the additional messages allow us to classify all the histories $h^{\tilde{\mathbb{T}}}$ into two large classes, depending on whether they are associated with the message $\mathfrak{m}_{0}$ or the message $\mathfrak{m}_{p}$. Recall that when the firm observes the message $\mathfrak{m}_{z}$, $z \in\{0, p\}$, its belief is equal to $z$. Thus, by the martingale property of the beliefs (see Aumann and Maschler 1995 and Kamenica and Gentzkow 2011), we conclude that the probability of observing the message $\mathfrak{m}_{0}$ is equal to $\left(1-\frac{p_{0}}{p}\right)$, while the probability of observing the message $\mathfrak{m}_{p}$ is equal $\frac{p_{0}}{p}$. Below, we use this fact to express the bounds on the continuation payoffs and the length of the relationship in terms of the endogenous variables $\Upsilon_{z}$ and $\tilde{q}_{z}, z \in\{0, p\}$.

First, observe that we can rewrite the firm's payoff $\bar{V}_{F}\left(h^{0}\right)$ as

$$
\begin{aligned}
\bar{V}_{F}\left(h^{0}\right)= & \left(1-\frac{p_{0}}{p}\right) \mathbb{E}_{(\sigma, \mu)}\left[(1-\delta) \sum_{t=0}^{\tilde{\mathbb{T}}-1} \delta^{t} \pi_{H}\left(q_{t}\right) \mid \mathfrak{m}_{0}\right] \\
& +\frac{p_{0}}{p} \mathbb{E}_{(\sigma, \mu)}\left[(1-\delta) \sum_{t=0}^{\tilde{\mathbb{T}}-1} \delta^{t} \pi_{H}\left(q_{t}\right)+\mathbb{I}_{\{\tilde{\mathbb{T}}<\infty\}} \delta^{\tilde{\mathbb{T}}} p \pi_{L}\left(q_{L}^{*}\right) \mid \mathfrak{m}_{p}\right]+K(1-\delta) .
\end{aligned}
$$

We now turn to the low type's payoffs. Fix an arbitrary outcome $\left(h^{\tilde{\mathbb{T}}}, \mathfrak{m}_{p}\right)$ and let $\operatorname{Pr}\left(h^{\tilde{\mathbb{T}}}, \mathfrak{m}_{p}\right)$ denote the ex ante probability of the outcome. Recall that the firm's belief upon observing the outcome $\left(h^{\tilde{\mathbb{T}}}, \mathfrak{m}_{p}\right)$ is equal to $p$. This immediately implies

$$
\operatorname{Pr}\left(h^{\tilde{\mathbb{T}}}, \mathfrak{m}_{p}\right)=\frac{p_{0}}{p} \operatorname{Pr}\left(h^{\tilde{\mathbb{T}}}, \mathfrak{m}_{p} \mid L\right)=\frac{1-p_{0}}{1-p} \operatorname{Pr}\left(h^{\tilde{\mathbb{T}}}, \mathfrak{m}_{p} \mid H\right) .
$$

We conclude that the outcome $\left(h^{\tilde{\mathbb{T}}}, \mathfrak{m}_{p}\right)$ is reached with probability $\frac{p}{p_{0}} \operatorname{Pr}\left(h^{\tilde{\mathbb{T}}}, \mathfrak{m}_{p}\right)$ when the worker announces that his type is low, and with probability $\frac{1-p}{1-p_{0}} \operatorname{Pr}\left(h^{\tilde{\mathbb{T}}}, \mathfrak{m}_{p}\right)$ when the worker announces that his type is high.

Similarly, an outcome $\left(h^{\tilde{\mathbb{T}}}, \mathfrak{m}_{0}\right)$ is reached with probability $\frac{1}{1-p_{0}} \operatorname{Pr}\left(h^{\tilde{\mathbb{T}}}, \mathfrak{m}_{0}\right)$ if the worker announces the high type and with probability 0 if the worker announces the low type $\left(\operatorname{Pr}\left(h^{\tilde{\mathbb{T}}}, \mathfrak{m}_{0}\right)\right.$ denotes the ex ante probability of the outcome).

Combining these observations, we can rewrite the low type's payoffs as

$$
\begin{aligned}
\bar{W}_{L}\left(h^{0}\right)= & \mathbb{E}_{(\sigma, \mu)}\left[(1-\delta) \sum_{t=0}^{\tilde{\mathbb{T}}-1} \delta^{t} \Delta \theta q_{t} \mid \mathfrak{m}_{p}\right]+K(1-\delta) \\
\underline{W}_{L H}\left(h^{0}\right)= & \left(\frac{1}{1-p_{0}}\right)\left(1-\frac{p_{0}}{p}\right) \mathbb{E}_{(\sigma, \mu)}\left[(1-\delta) \sum_{t=0}^{\tilde{\mathbb{T}}-1} \delta^{t} \Delta \theta q_{t} \mid \mathfrak{m}_{0}\right] \\
& +\left(\frac{1-p}{1-p_{0}}\right)\left(\frac{p_{0}}{p}\right) \mathbb{E}_{(\sigma, \mu)}\left[(1-\delta) \sum_{t=0}^{\tilde{\mathbb{T}}-1} \delta^{t} \Delta \theta q_{t} \mid \mathfrak{m}_{p}\right]-K(1-\delta) .
\end{aligned}
$$


We next provide the formal definitions of $\Upsilon_{z}$ and $\tilde{q}_{z}$ for $z \in\{0, p\}$ :

$$
\Upsilon_{z}=\mathbb{E}_{(\sigma, \mu)}\left[(1-\delta) \sum_{t=0}^{\tilde{\mathbb{T}}-1} \delta^{t} \mid \mathfrak{m}_{z}\right]
$$

and

$$
\tilde{q}_{z}=\frac{1}{\Upsilon_{z}} \mathbb{E}_{(\sigma, \mu)}\left[(1-\delta) \sum_{t=0}^{\tilde{\mathbb{T}}-1} \delta^{t} q_{t} \mid \mathfrak{m}_{z}\right]
$$

if $\Upsilon_{z} \neq 0$, and $\tilde{q}_{z}=0$ otherwise.

The definitions of $Y_{z}$ and $\tilde{q}_{z}, z \in\{0, p\}$, allow us to express $\bar{W}_{L}\left(h^{0}\right)$ and $\underline{W}_{L H}\left(h^{0}\right)$ as in (5). The inequality $\bar{V}_{F}\left(h^{0}\right) \leq \breve{V}_{F}\left(h^{0}\right)$ in (5) follows from (7), the concavity of the function $\pi_{H}(\cdot)$, and Jensen's inequality.

Finally, using the definitions of $\Upsilon_{0}$ and $\Upsilon_{p}$ and inequality (4), we are able to bound the length of the high type's relationship as in (6).

Step 4: Bounding the expected length of the high type's relationship. The following claim establishes the first property of a firing region.

Claim 1. Fix $K>0$ and $p>\hat{p}$. There exists $K^{\prime}>0$ such that, for every $p_{0} \in\left[p-\frac{f(p)}{2}, p\right]$, for every $\delta$, and for every $\left(\Upsilon_{z}, \tilde{q}_{z}\right) \in[0,1]^{2}, z \in\{0, p\}$, the inequalities

$$
\begin{aligned}
\left(1-\frac{p_{0}}{p}\right) \Upsilon_{0} \pi_{H}\left(\tilde{q}_{0}\right)+ & \frac{p_{0}}{p}\left[\Upsilon_{p} \pi_{H}\left(\tilde{q}_{p}\right)+\left(1-\Upsilon_{p}\right) p \pi_{L}\left(q_{L}^{*}\right)\right]+K(1-\delta) \geq p_{0} \pi_{L}\left(q_{L}^{*}\right) \\
\Upsilon_{p} \tilde{q}_{p} \Delta \theta+K(1-\delta) \geq & \left(\frac{1}{1-p_{0}}\right)\left(1-\frac{p_{0}}{p}\right) \Upsilon_{0} \tilde{q}_{0} \Delta \theta \\
& +\left(\frac{1-p}{1-p_{0}}\right)\left(\frac{p_{0}}{p}\right) \Upsilon_{p} \tilde{q}_{p} \Delta \theta-K(1-\delta)
\end{aligned}
$$

are simultaneously satisfied only if

$$
\left(\frac{1}{1-p_{0}}\right)\left(1-\frac{p_{0}}{p}\right) \Upsilon_{0}+\left(\frac{1-p}{1-p_{0}}\right)\left(\frac{p_{0}}{p}\right) \Upsilon_{p}+K(1-\delta) \leq K^{\prime}(1-\delta) .
$$

The inequalities (8) and (9) capture the constraints $\breve{V}_{F}\left(h^{0}\right) \geq p_{0} \pi_{L}\left(q_{L}^{*}\right)$ and $\bar{W}_{L}\left(h^{0}\right) \geq$ $\underline{W}_{L H}\left(h^{0}\right)$, respectively, while the left hand side of inequality (10) represents the upper bound to the expected discounted length of the high type's relationship.

The proof of Claim 1 is tedious and is relegated to the Appendix. The logic behind this claim is better understood when one considers the problem of maximizing $\breve{V}_{F}\left(h^{0}\right)$ (with respect to $Y_{z}$ and $\tilde{q}_{z}, z \in\{0, p\}$ ) subject to the low type's incentive compatibility constraint: $\bar{W}_{L}\left(h^{0}\right) \geq \underline{W}_{L H}\left(h^{0}\right)$. Clearly, $\breve{V}_{F}\left(h^{0}\right)$ is maximized by setting $\Upsilon_{0}$ equal to $1, \tilde{q}_{0}$ equal to $q_{H}^{*}$, and $\Upsilon_{p}$ equal to 0 (recall that $p>\hat{p}$ and, therefore, $p \pi_{L}\left(q_{L}^{*}\right)>\pi_{H}\left(q_{H}^{*}\right) \geq$ $\pi_{H}\left(\tilde{q}_{p}\right)$ for any $\left.\tilde{q}_{p}\right)$. However, this would violate the low type's incentive compatibility constraint. Hence, the following trade-off emerges. To increase the firm's payoff by increasing $Y_{0}$ while also satisfying the incentive compatibility constraint (9), it is also 
necessary to increase $\Upsilon_{p}$, which decreases the firm's payoff. Notice that when the prior $p_{0}$ is close to $p,\left(1-\frac{p_{0}}{p}\right) \Upsilon_{0}$ and $\tilde{q}_{0}$ have a small impact on both the firm's payoff and the constraint. In contrast, $\Upsilon_{p}$ has a small impact on the constraint and a large (negative) impact on the firm's payoff. We conclude that for $\delta$ and $p_{0}$ sufficiently large, the optimal values of $\left(1-\frac{p_{0}}{p}\right) \Upsilon_{0}$ and $\Upsilon_{p}$ must be close to 0 . Therefore, if we could maximize the firm's payoff, subject to $\bar{W}_{L}\left(h^{0}\right) \geq \underline{W}_{L H}\left(h^{0}\right)$, the solution would be close to a firing allocation, yielding a payoff close to $p_{0} \pi_{L}\left(q_{L}^{*}\right)$. For the same reason, any allocation that satisfies $\bar{W}_{L}\left(h^{0}\right) \geq \underline{W}_{L H}\left(h^{0}\right)$ and that is not close to a firing allocation leads to a payoff for the firm smaller than $p_{0} \pi_{L}\left(q_{L}^{*}\right)$, hence, violating (8).

We have shown that the first property of a firing region holds. In particular, there exist $\bar{K}>0$ and $\bar{\delta}<1$ such that for any $\delta>\bar{\delta}$, any prior $p_{0}$ above $p-\frac{f(p)}{2}$, and any PBE $(\sigma, \mu)$, we have $E_{(\sigma, \mu)}\left[(1-\delta) \sum_{t=0}^{\mathbb{T}-1} \delta^{t} \mid h^{0}, H\right] \leq(1-\delta) \bar{K}$. We now turn to the remaining two properties. To verify the second property (the firm's payoff conditional on type $H$ shrinks to 0 weakly faster than $1-\delta$ ), notice that

$$
V_{F}\left(h^{0} ; H\right) \leq v(1) \mathbb{E}_{(\sigma, \mu)}\left[(1-\delta) \sum_{t=0}^{\mathbb{T}-1} \delta^{t} \mid h^{0}, H\right] \leq v(1) \bar{K}(1-\delta) .
$$

Finally, we use the result above to bound the low type's continuation payoff $W_{L}\left(h^{0}\right)$ (third property). We have

$$
p_{0} \pi_{L}\left(q_{L}^{*}\right) \leq V_{F}\left(h^{0}\right) \leq\left(1-p_{0}\right) V_{F}\left(h^{0} ; H\right)+p_{0}\left(\pi_{L}\left(q_{L}^{*}\right)-W_{L}\left(h^{0}\right)\right),
$$

which implies

$$
W_{L}\left(h^{0}\right) \leq \frac{1-p_{0}}{p_{0}} V_{F}\left(h^{0} ; H\right)<\frac{1-\hat{p}}{\hat{p}} V_{F}\left(h^{0} ; H\right) \leq \frac{1-\hat{p}}{\hat{p}} v(1) \bar{K}(1-\delta) .
$$

This concludes the proof of Lemma 5.

\subsection{Low belief case: $p<\hat{p}$}

In this section, we characterize the limiting equilibrium outcome when the prior is lower than $\hat{p}$. To do so, we first define the notion of pooling region.

Definition 3. The interval $[0, p]$ is a pooling region if, for every $\varepsilon>0$, there exists $\bar{\delta}<1$ such that the following statement holds: Fix $\delta>\bar{\delta}$, an arbitrary PBE $(\sigma, \mu)$, and a history $h^{t}$ at which $\mu\left(h^{t}\right) \leq p$. Then we have

(i) $\mathbb{E}_{(\sigma, \mu)}\left[\delta^{\mathbb{T}}\right]<\varepsilon$

(ii) $\mathbb{E}_{(\sigma, \mu)}\left[(1-\delta) \sum_{t=0}^{\mathbb{T}-1} \delta^{t}\left|q_{t}-q_{H}^{*}\right|\right]<\varepsilon$

(iii) For $i \in\{H, L\}, \mathbb{E}_{(\sigma, \mu)}\left[(1-\delta) \sum_{t=0}^{\mathbb{T}-1} \delta^{t}\left(x_{t}-\theta_{H} q_{H}^{*}-\alpha\right) \mid i\right]<\varepsilon$.

When the belief is in a pooling region, the equilibrium allocation converges to the pooling allocation as $\delta$ goes to 1 . Our next result shows that all beliefs lower than $\hat{p}$ belong to a pooling region. 
Proposition 3. For every $p<\hat{p}$, the interval $[0, p]$ is a pooling region.

Before turning to the proof of Proposition 3, we establish a preliminary result. We show that the equilibrium belief cannot grow too quickly around $\hat{p}$ when the parties are sufficiently patient.

Lemma 6. For every $\varepsilon>0$, there exists $\bar{\delta}<1$ such that the following statement holds: Fix $\delta>\bar{\delta}$ and a $\operatorname{PBE}(\sigma, \mu)$. There does not exist a history $h^{t}$ with $\mu\left(h^{t}\right)<\hat{p}-\varepsilon$ at which the firm offers a menu $m_{t}$ that contains a contract $\left(x_{t}, q_{t}\right)$ accepted with positive probability and such that $\mu\left(h^{t}, m_{t},\left(x_{t}, q_{t}\right)\right)>\hat{p}+\varepsilon$.

The proof of Lemma 6 is given in Appendix B. Recall that for every $\varepsilon>0$, the interval $[\hat{p}+\varepsilon, 1]$ is a firing region. Therefore, it follows from Lemma 4 that if $\delta$ is close to 1 and the belief jumps from $\mu\left(h^{t}\right)<\hat{p}-\varepsilon$ to $\mu\left(h^{t}, m_{t},\left(x_{t}, q_{t}\right)\right)>\hat{p}+\varepsilon$, the firm's continuation payoff at $h^{t}$ must be close to $\mu\left(h^{t}\right) \pi_{L}\left(q_{L}^{*}\right)$. But then the firm's payoff would be smaller than $\pi_{H}\left(q_{H}^{*}\right)$ (since $\mu\left(h^{t}\right)<\hat{p}-\varepsilon$ and $\hat{p} \pi_{L}\left(q_{L}^{*}\right)=\pi_{H}\left(q_{H}^{*}\right)$ ), contradicting Lemma 1.

We now outline the proof of Proposition 3 (see Appendix B for the formal proof). As in the previous section, we simplify the notation and establish the three properties of a pooling region for the initial history $h^{0}$.

The first two properties do not depend on equilibrium transfers. Therefore, it is without loss of generality to establish these properties for the class of equilibria in which (a) the firm's strategy in the first period is pure; and (b) the high type's equilibrium payoff is equal to $0 .^{20}$

We start with the first property and fix $p<\hat{p}$. By contradiction, let us assume that there exists a sequence $\left\{\delta_{n}, p_{0, n},\left(\sigma_{n}, \mu_{n}\right)\right\}_{n=1}^{\infty}$ such that $\delta_{n}$ converges to $1, p_{0, n} \in[0, p]$, $\left(\sigma_{n}, \mu_{n}\right)$ is a PBE of the game with discount factor equal to $\delta_{n}$ and prior equal to $p_{0, n}$, and $\lim _{n \rightarrow \infty} \mathbb{E}_{\left(\sigma_{n}, \mu_{n}\right)}\left[\delta_{n}^{\mathbb{T}}\right]=\xi>0$. We show that for $n$ sufficiently large, it is strictly profitable for the low type to deviate from the equilibrium strategy and mimic the high type. To ease the notation, in what follows we suppress the index $n$ and write $\delta, p_{0}$, and $(\sigma, \mu)$ to denote an arbitrary element of the sequence.

Below, we proceed as follows. First, we replace the low type's payoffs (from the equilibrium strategy and the deviation) with bounds that depend on the expected discounted time at which the firm's belief falls for the first time in the firing region. We then compute this expected discounted time conditional on the worker's type. With this we show that when the parties are sufficiently patient, it is strictly profitable for the low type to deviate and mimic the high type.

Fix a small $\varepsilon$ and now let $\tilde{\mathbb{T}} \in \mathbf{N} \cup\{\infty\}$ denote the random time that stops the play at the first history $\left(h^{\tilde{\mathbb{T}}}, m_{\tilde{\mathbb{T}}}\right)$ at which the menu $m_{\tilde{\mathbb{T}}}$ contains a contract $\left(x_{\tilde{\mathbb{T}}}, q_{\tilde{\mathbb{T}}}\right)$ that is accepted with a positive probability and for which $\mu\left(h^{\tilde{\mathbb{T}}}, m_{\tilde{\mathbb{T}}},\left(x_{\tilde{\mathbb{T}}}, q_{\tilde{\mathbb{T}}}\right)\right) \geq \hat{p}+\varepsilon$. Recall that $[\hat{p}+\varepsilon, 1]$ is a firing region and $W_{H}\left(h^{0}\right)=0$. We proceed similarly as in Section 5.1 by making a change in the timing of the transfers and using Lemma 4 to bound the

\footnotetext{
${ }^{20}$ The proof of this claim is identical to the proof provided in Section 5.1 and, therefore, is omitted.
} 
continuation payoffs at $\left(h^{\tilde{\mathbb{T}}}, m_{\tilde{\mathbb{T}}}\right.$ ) (see the discussion after inequality (4)). For $\delta$ close to 1 , the low type's payoffs $W_{L}\left(h^{0}\right)$ and $W_{L H}\left(h^{0}\right)$ are bounded as

$$
\begin{aligned}
W_{L}\left(h^{0}\right) \leq \mathbb{E}_{(\sigma, \mu)}\left[(1-\delta) \sum_{t=0}^{\tilde{\mathbb{T}}-1} \delta^{t} \Delta \theta q_{t} \mid L\right]+\varepsilon \\
W_{L H}\left(h^{0}\right) \geq \mathbb{E}_{(\sigma, \mu)}\left[(1-\delta) \sum_{t=0}^{\tilde{\mathbb{T}}-1} \delta^{t} \Delta \theta q_{t} \mid H\right]-\varepsilon .
\end{aligned}
$$

Also, for $\delta$ close to 1 , the firm's payoff is bounded by

$$
\begin{aligned}
V_{F}\left(h^{0}\right) & \leq \mathbb{E}_{(\sigma, \mu)}\left[(1-\delta) \sum_{t=0}^{\tilde{\mathbb{T}}-1} \delta^{t} \pi_{H}\left(q_{t}\right)+\mathbb{I}_{\{\tilde{\mathbb{T}}<\infty\}} \delta^{\tilde{\mathbb{T}}} \mu\left(h^{\tilde{\mathbb{T}}}\right) \pi_{L}\left(q_{L}^{*}\right)\right]+\varepsilon \\
& \leq \mathbb{E}_{(\sigma, \mu)}\left[(1-\delta) \sum_{t=0}^{\tilde{\mathbb{T}}-1} \delta^{t} \pi_{H}\left(q_{t}\right)+\mathbb{I}_{\{\tilde{\mathbb{T}}<\infty\}} \delta^{\tilde{\mathbb{T}}}(\hat{p}+\varepsilon) \pi_{L}\left(q_{L}^{*}\right)\right]+\varepsilon,
\end{aligned}
$$

where the second inequality holds because the belief at the history $h^{\tilde{\mathbb{T}}}$ is bounded above (by definition) by $\hat{p}+\varepsilon$.

Notice that $\varepsilon$ can be arbitrarily small. Therefore, since $\pi_{H}\left(q_{H}^{*}\right)=\hat{p} \pi_{L}\left(q_{L}^{*}\right)$ and $q_{H}^{*}$ is the unique maximizer of $\pi_{H}(\cdot)$, the inequality above implies that

$$
\mathbb{E}_{(\sigma, \mu)}\left[(1-\delta) \sum_{t=0}^{\tilde{\mathbb{T}}-1} \delta^{t}\left|q_{H}^{*}-q_{t}\right|\right] \approx 0
$$

for $\delta$ sufficiently large. If this were not the case, then the firm's payoff would be strictly smaller than $\pi_{H}\left(q_{H}^{*}\right)$ (again, for $\delta$ sufficiently large).

Combining (11) with (12), we obtain that for $\delta$ close to 1 , the upper bound of $W_{L}\left(h^{0}\right)$ is close to

$$
\Delta \theta q_{H}^{*} \mathbb{E}_{(\sigma, \mu)}\left[(1-\delta) \sum_{t=0}^{\tilde{\mathbb{T}}-1} \delta^{t} \mid L\right]=\Delta \theta q_{H}^{*}\left(1-\mathbb{E}_{(\sigma, \mu)}\left[\delta^{\tilde{\mathbb{T}}} \mid L\right]\right),
$$

while the lower bound of $W_{L H}\left(h^{0}\right)$ is close to

$$
\Delta \theta q_{H}^{*} \mathbb{E}_{(\sigma, \mu)}\left[(1-\delta) \sum_{t=0}^{\tilde{\mathbb{T}}-1} \delta^{t} \mid H\right]=\Delta \theta q_{H}^{*}\left(1-\mathbb{E}_{(\sigma, \mu)}\left[\delta^{\tilde{\mathbb{T}}} \mid H\right]\right)
$$

We now approximate $\mathbb{E}_{(\sigma, \mu)}\left[\delta^{\tilde{\mathbb{T}}} \mid L\right]$ and $\mathbb{E}_{(\sigma, \mu)}\left[\delta^{\tilde{\mathbb{T}}} \mid H\right]$. We do this in two steps. First, we express $\mathbb{E}_{(\sigma, \mu)}\left[\delta^{\tilde{\mathbb{T}}} \mid i\right]$ as function of $\mathbb{E}_{(\sigma, \mu)}\left[\delta^{\tilde{\mathbb{T}}}\right]$. Then we approximate $\mathbb{E}_{(\sigma, \mu)}\left[\delta^{\tilde{\mathbb{T}}}\right]$.

Let $\operatorname{Pr}\left(h^{\tilde{\mathbb{T}}}\right)$ denote the (ex ante) probability of reaching the history $h^{\tilde{\mathbb{T}}}$. Then $h^{\tilde{\mathbb{T}}}$ is reached with a probability $\frac{\mu\left(h^{\tilde{\mathbb{T}}}\right)}{p_{0}} \operatorname{Pr}\left(h^{\tilde{\mathbb{T}}}\right)$ if the low type follows his strategy $\sigma^{L}$, and is 
reached with a probability $\frac{1-\mu\left(h^{\tilde{T}}\right)}{1-p_{0}} \operatorname{Pr}\left(h^{\tilde{\mathbb{T}}}\right)$ if he mimics the high type and plays the strategy $\sigma^{H}$. Also, it follows from Lemma 6 that for $\delta$ close to $1, \mu\left(h^{\tilde{\mathbb{T}}}\right)$ must be close to $\hat{p}$. Therefore, for $\delta$ sufficiently large, we have

$$
\begin{aligned}
\mathbb{E}_{(\sigma, \mu)}\left[\delta^{\tilde{\mathbb{T}}} \mid L\right] & \approx \frac{\hat{p}}{p_{0}} \mathbb{E}_{(\sigma, \mu)}\left[\delta^{\tilde{\mathbb{T}}}\right] \\
\mathbb{E}_{(\sigma, \mu)}\left[\delta^{\tilde{\mathbb{T}}} \mid H\right] & \approx \frac{1-\hat{p}}{1-p_{0}} \mathbb{E}_{(\sigma, \mu)}\left[\delta^{\tilde{\mathbb{T}}}\right] .
\end{aligned}
$$

Next, we examine the relationship between $\mathbb{E}_{(\sigma, \mu)}\left[\delta^{\mathbb{T}}\right]$ and $\mathbb{E}_{(\sigma, \mu)}\left[\delta^{\tilde{\mathbb{T}}}\right]$ when $\delta$ is close to 1 . First, at the history $\left(h^{\tilde{\mathbb{T}}}, m_{\tilde{\mathbb{T}}}\right)$, the expected discounted length of the relationship with the high type is close to 0 (see Lemma 4 and recall that the interval $[\hat{p}+\varepsilon, 1]$ is a firing region). Second, the firm's belief at $h^{\tilde{\mathbb{T}}}$ must be close to $\hat{p}$ (a value of $\mu\left(h^{\tilde{\mathbb{T}}}\right)$ far away from $\hat{p}$ would contradict Lemma 6). Finally, recall that for $\delta$ large, $\mathbb{E}_{(\sigma, \mu)}\left[\delta^{\mathbb{T}}\right]$ is close (by assumption) to $\xi$. Putting these observations together and using Bayes' rule, we conclude that $\mathbb{E}_{(\sigma, \mu)}\left[\delta^{\widetilde{T}}\right]$ is close to $\frac{\xi}{1-\hat{p}}$ for $\delta$ close to 1 .

Combining (13), (14), (15), and the last observation, we conclude that for $\delta$ close to 1 , the upper bound of $W_{L}\left(h^{0}\right)$ is close to

$$
\Delta \theta q_{H}^{*}\left(1-\frac{\hat{p}}{p_{0}} \frac{\xi}{1-\hat{p}}\right) \leq \Delta \theta q_{H}^{*}\left(1-\frac{\hat{p}}{p} \frac{\xi}{1-\hat{p}}\right)
$$

(where the inequality follows from $p_{0} \leq p$ ), while the lower bound of $W_{L H}\left(h^{0}\right)$ is close to

$$
\Delta \theta q_{H}^{*}\left(1-\frac{1-\hat{p}}{1-p_{0}} \frac{\xi}{1-\hat{p}}\right) \geq \Delta \theta q_{H}^{*}\left(1-\frac{1-\hat{p}}{1-p} \frac{\xi}{1-\hat{p}}\right) .
$$

Hence, since $p<\hat{p}$ and $\xi>0$, we have

$$
\Delta \theta q_{H}^{*}\left(1-\frac{\hat{p}}{p} \frac{\xi}{1-\hat{p}}\right)<\Delta \theta q_{H}^{*}\left(1-\frac{1-\hat{p}}{1-p} \frac{\xi}{1-\hat{p}}\right),
$$

which implies the existence of a profitable deviation for values of $\delta$ close to 1 .

Finally, the second and third properties of a pooling region are direct consequences of the first property. Intuitively, if the high type never quits the relationship, the best option for the firm is to implement the best pooling allocation.

\section{REHIRING}

In the model analyzed so far, the worker's decision to reject all the contracts in the menu is an irreversible action that ends the relationship. In other words, the firm cannot rehire the worker after a period of unemployment. As we argued in the previous two sections, this impairs the firm's ability to screen the worker. Once the worker reveals his type, his continuation payoff must be equal to 0 . The firm can afford to pay the reservation wage, because the worker has no alternative but to end the relationship.

This logic does not apply when rehiring is possible. In this case, the worker can credibly threaten the firm to reject offers that pay slightly above the reservation wage, 
because he expects to obtain a large payoff in the rest of the relationship. As we see below, with rehiring, it is possible to sustain equilibrium outcomes in which the worker's payoff remains strictly positive even when his type is known to the other party. This, in turn, makes it easier for the firm to screen the worker.

There are different ways to break the link between the decision to reject all the contracts and the decision to end the relationship. One possibility is to assume that the relationship lasts forever and quitting is not allowed. Another possibility is to add to the benchmark model the option for the worker to reject all the contracts and remain in the relationship. In the rest of this section, we analyze these extensions of the model.

We start with the infinitely repeated game in which, in each period, the firm proposes a menu of contracts. The worker either accepts a contract in the menu or rejects all of them. Both parties obtain a payoff equal to 0 in each period in which the worker rejects all the contracts in the menu.

Consider the standard mechanism design problem with commitment. We say that the payoffs $\left(V_{F, H}, V_{F, L}, W_{H}, W_{L}\right)$ are incentive-compatible and ex post strictly individually rational if there exists an incentive-compatible direct mechanism $\left\{\left(x_{H}, q_{H}\right)\right.$, $\left.\left(x_{L}, q_{L}\right)\right\},\left(x_{i}, q_{i}\right) \in \mathbb{R}_{++} \times[0,1]$ for $i \in\{H, L\}$, that satisfies the following statements:

(i) For $i \in\{H, L\}$, the firm's payoff $V_{F, i}$ when the worker is of type $i$ is strictly positive: $V_{F, i}:=v\left(q_{i}\right)-x_{i}>0$.

(ii) For $i \in\{H, L\}$, type $i$ 's payoff is strictly positive: $W_{i}:=x_{i}-\theta_{i} q_{i}-\alpha>0$.

The main result of this section is a folk theorem. We show that any profile of incentive-compatible and ex post strictly individual rational payoffs can be achieved in the infinitely repeated game when the parties are sufficiently patient.

Proposition 4. For every tuple $\left(V_{F, H}, V_{F, L}, W_{H}, W_{L}\right) \in \mathbb{R}_{++}^{4}$ of incentive-compatible and ex post strictly individually rational payoffs there exists $\delta^{\dagger} \in(0,1)$ such that for every $\delta \geq \delta^{\dagger}$ there exists a PBE (of the infinitely repeated game) that leads to such payoffs.

The proof of Proposition 4 is given in the Supplemental Material. Fix a tuple $\left(V_{F, H}, V_{F, L}, W_{H}, W_{L}\right)$ of incentive-compatible and ex post strictly individually rational payoffs and let $\left\{\left(x_{H}, q_{H}\right),\left(x_{L}, q_{L}\right)\right\}$ denote the corresponding direct mechanism. We construct an equilibrium that consists of two phases. The screening phase takes place in the first period when the firm offers the menu $\left\{\left(x_{H}, q_{H}\right),\left(x_{L}, q_{L}\right)\right\}$. Each type $i \in\{H, L\}$ selects the menu $\left(x_{i}, q_{i}\right)$ and the firm learns the worker's type. The post-screening phase with type $i \in\{H, L\}$ begins in the second period and implements the contract $\left(x_{i}, q_{i}\right)$ in every period.

In equilibrium, the firm never updates its belief in the post-screening phase. It is, therefore, necessary to show that in the game with complete information with type $i$, there exists an equilibrium that implements $\left(x_{i}, q_{i}\right)$ in every period (when the parties are sufficiently patient). The following lemma establishes this important result.

Lemma 7. Consider the infinitely repeated game with complete information in which the firm interacts with type $i \in\{H, L\}$. Let $\left(x_{i}, q_{i}\right)$ be a contract that yields the payoff $V_{F, i}=$ 
$v\left(q_{i}\right)-x_{i}>0$ to the firm and the payoff $W_{i}=x_{i}-\theta_{i} q_{i}-\alpha>0$ to the worker. There exists $\delta^{\dagger} \in(0,1)$ such that for every $\delta \geq \delta^{\dagger}$, there exists a subgame perfect equilibrium that leads to the payoffs $\left(V_{F, i}, W_{i}\right)$.

Proof. Fix $\varepsilon \in\left(0, \min \left\{V_{F, i}, W_{i}\right\}\right)$. Let $\left(\underline{x}_{i}, q_{i}^{*}\right), \underline{x}_{i}=\alpha+\theta_{i} q_{i}^{*}+\frac{\varepsilon}{2}$, denote the efficient contract that yields the payoff $\frac{\varepsilon}{2}$ to the worker. Also, let $\left(\bar{x}_{i}, q_{i}^{*}\right), \bar{x}_{i}=v\left(q_{i}^{*}\right)-\frac{\varepsilon}{2}$, denote the efficient contract that yields the payoff $\frac{\varepsilon}{2}$ to the firm.

Consider the following strategy profile, generated by a simple three state automaton.

State $(i, 0)$. This is the initial state. The automaton prescribes that the firm offers the menu $\left\{\left(x_{i}, q_{i}\right)\right\}$ and the worker accepts the contract $\left(x_{i}, q_{i}\right)$. The state remains $(i, 0)$ unless there is a deviation by the firm, in which case the state changes to $(i, 1)$, irrespective of the worker's decision. When the firm deviates and offers a menu different from $\left\{\left(x_{i}, q_{i}\right)\right\}$, the worker accepts the contract that maximizes his current payoff, provided that this is positive (here and in what follows, we require the worker to select the contract with the smallest index if there are multiple contracts that yield the highest current payoff). Finally, the worker rejects all the contracts if they all yield a negative payoff.

State $(i, 1)$. The automaton prescribes that the firm offers the menu $\left\{\left(\bar{x}_{i}, q_{i}^{*}\right)\right\}$ and the worker accepts $\left(\bar{x}_{i}, q_{i}^{*}\right)$. If the firm offers $\left\{\left(\bar{x}_{i}, q_{i}^{*}\right)\right\}$, the state remains $(i, 1)$ irrespective of the worker's decision. Suppose instead that the firm deviates and offers the menu $m \neq$ $\left\{\left(\bar{x}_{i}, q_{i}^{*}\right)\right\}$. The worker rejects every contract $(x, q)$ with $x<v(1)+\alpha$ and selects, among the remaining contracts, the contract that yields the highest current payoff, provided that this is positive. If the worker accepts a contract $(x, q)$ with $x<v(1)+\alpha$, the state changes to $(i, 2)$. In all other cases, the state remains $(i, 1)$.

State $(i, 2)$. The automaton prescribes that the firm offers the menu $\left\{\left(\underline{x}_{i}, q_{i}^{*}\right)\right\}$ and the worker accepts $\left(\underline{x}_{i}, q_{i}^{*}\right)$. The state remains $(i, 2)$ unless there is a deviation by the firm. In this case, the state changes to $(i, 1)$, irrespective of the worker's decision. When the firm deviates and offers a menu different from $\left\{\left(\underline{x}_{i}, q_{i}^{*}\right)\right\}$, the worker accepts the contract that maximizes his current payoff, provided that this is positive.

This concludes the description of the automaton. We now verify that this strategy profile is a subgame perfect equilibrium for values of $\delta$ sufficiently large. The firm's equilibrium payoffs are $V_{F, i}$ in state $(i, 0), \frac{\varepsilon}{2}$ in state $(i, 1)$, and $v\left(q_{i}^{*}\right)-\underline{x}_{i}$ in state $(i, 2)$. The worker's equilibrium payoffs are $W_{i}$ in state $(i, 0), \bar{x}_{i}-\theta_{i} q_{i}^{*}-\alpha$ in state $(i, 1)$, and $\frac{\varepsilon}{2}$ in state $(i, 2)$. Thus, the firm obtains the largest payoff in state $(i, 2)$ and the lowest payoff in state $(i, 1)$, while the worker obtains the largest payoff in state $(i, 1)$ and the lowest payoff in state $(i, 2)$. In fact, notice that $V_{F, i}+W_{i}=\pi_{i}\left(q_{i}\right)$ and $\pi_{i}\left(q_{i}^{*}\right) \geq \pi_{i}\left(q_{i}\right)$ for every $q_{i}$. This and the fact that $\varepsilon \in\left(0, \min \left\{V_{F, i}, W_{i}\right\}\right)$ imply

$$
\begin{gathered}
v\left(q_{i}^{*}\right)-\underline{x}_{i}>V_{F, i}>v\left(q_{i}^{*}\right)-\bar{x}_{i}=\frac{\varepsilon}{2} \\
\bar{x}_{i}-\theta_{i} q_{i}^{*}-\alpha>W_{i}>\underline{x}_{i}-\theta_{i} q_{i}^{*}-\alpha=\frac{\varepsilon}{2} .
\end{gathered}
$$

Thus, for $\delta$ sufficiently large, one-shot deviations from the automaton described above are not profitable. 
Recall that the mechanism $\left\{\left(x_{H}, q_{H}\right),\left(x_{L}, q_{L}\right)\right\}$ is incentive-compatible. Thus, if the post-screening phase with type $i \in\{H, L\}$ implements the contract $\left(x_{i}, q_{i}\right)$ in every period, then in the screening phase, both types of the worker have an incentive to reveal their type. The rest of the proof of Proposition 4 provides a complete description of the equilibrium strategies and beliefs, and verifies that sequential rationality is satisfied at all histories.

Consider now a different extension of the benchmark model in which the worker has the option to reject all the contracts and remain in the relationship. The result of Proposition 4 extends to this setup. To see this, notice that the strategy of rejecting all the contracts and quitting is weakly dominated by the strategy of rejecting all the contracts and remaining in the relationship. Once we remove the strategy of quitting, we are back to the infinitely repeated game analyzed above.

In summary, our analysis shows that when the relationship can continue after the worker rejects all the contracts (either because the game is infinitely repeated or because the worker has the option to reject all the contracts and stay in the relationship), many screening opportunities are available to the firm, including separation with employment. This is in contrast to the benchmark model in which the relationship ends automatically after the worker rejects all the contracts. In this case, only the pooling and the firing allocations can be implemented when the parties are sufficiently patient.

\section{CONCLUding REMARKS}

We study a dynamic-contracting model with adverse selection and limited commitment. In our benchmark model, the relationship ends when the worker rejects all the contracts from the firm's menu. We characterize the limiting equilibrium outcome as the parties become arbitrarily patient. If the prior probability that the worker has a low cost is low, the firm offers a pooling contract in every period. In contrast, if the prior is high, the firm fires the high-cost worker at the beginning of the relationship.

We conduct our analysis under the assumption that the worker bears a cost $\alpha>0$ when he is employed. The assumption simplifies the analysis (it is easier to link the expected length of the relationship with the expected quality of the good provided by the worker), but is not crucial. In fact, all our results extend to the case in which $\alpha$ is equal to $0 .{ }^{21}$

In this paper, the worker's action is verifiable. In some situations, the agent's effort leads to stochastic outcomes and monitoring is thus imperfect. This adds a moral hazard component to the screening problem. We leave this interesting extension for future research.

\section{Appendix A: Proof of Proposition 1}

In this appendix, we prove the existence of PBE for generic values of the parameters. In particular, we construct an equilibrium in which the high type's payoff is equal to 0 .

\footnotetext{
${ }^{21}$ See the working paper at https://www.carloalberto.org/wp-content/uploads/2015/02/no.401.pdf.
} 
Although not Markovian, in our PBE, the firm and the low type's equilibrium continuation payoffs depend on the firm's belief. We use the function $V:[0,1] \rightarrow \mathbb{R}_{+}$to denote the firm's payoff (as a function of its belief) and the correspondence $\Phi:[0,1] \rightrightarrows \mathbb{R}_{+}$to denote the set of payoffs of the low type. To simplify the notation, we write $\Phi(p)=z$ for $\Phi(p)=\{z\}$. We also write $\min \Phi(p)(\max \Phi(p))$ to denote the smallest (largest) element of $\Phi(p)$.

For $\delta>\hat{\delta}$, we define $V$ and $\Phi$ as

$$
\begin{aligned}
& V(p)=\max \left\{\pi_{H}\left(q_{H}^{*}\right), p \pi_{L}\left(q_{L}^{*}\right)\right\} \\
& \Phi(p)= \begin{cases}\Delta \theta q_{H}^{*} & \text { for } p<\hat{p} \\
{\left[0, \Delta \theta q_{H}^{*}\right]} & \text { for } p=\hat{p} \\
0 & \text { for } p>\hat{p},\end{cases}
\end{aligned}
$$

where $\hat{p}$ satisfies $\pi_{H}\left(q_{H}^{*}\right)=\hat{p} \pi_{L}\left(q_{L}^{*}\right)$.

Next, we show that for generic values of $\delta$ smaller than $\hat{\delta}$, there exists a pair $(V, \Phi)$ that satisfies a number of properties.

Lemma 8. Fix the parameters $\left(\theta_{H}, \theta_{L}, \alpha, v(\cdot)\right)$. For all but at most two values of $\delta$ in $(0, \hat{\delta}]$, there exists a pair $(V, \Phi)$ that satisfies the following conditions:

(i) The function $V$ is continuous and the correspondence $\Phi$ is upper hemicontinuous.

(ii) There exists $\bar{p} \in(0,1)$ such that $V(p)=p \pi_{L}^{*}$ and $\Phi(p)=0$ for $p>\bar{p}$.

(iii) There exists $\underline{p} \in[0, \bar{p}]$ such that $V(p)=\pi_{H}^{*}$ for $p \leq \underline{p}$, $\Phi(p)=\Delta \theta q_{H}^{*}$ for $p<\underline{p}$, and $\Delta \theta q_{H}^{*}=\max \Phi(\underline{p})$.

(iv) We have $V(p)=\tilde{V}(p)$ for $p \in[\underline{p}, \bar{p}]$ and $V(p)>\tilde{V}(p)$ for $p \in(0, \underline{p}) \cup(\bar{p}, 1)$, where $\tilde{V}(p)$ is defined by

$$
\begin{aligned}
& \begin{array}{l}
\tilde{V}(p)= \\
\max _{\left(q_{H}, q_{L}\right) \in[0,1]^{2}, x \in \mathbb{R}, \tilde{p} \leq p} \frac{1-p}{1-\tilde{p}}\left[(1-\delta) \pi_{H}\left(q_{H}\right)+\delta V(\tilde{p})\right] \\
+\frac{p-\tilde{p}}{1-\tilde{p}}\left[(1-\delta)\left(v\left(q_{L}\right)-x\right)+\delta \pi_{L}\left(q_{L}^{*}\right)\right]
\end{array} \\
& \text { subject to } x-\theta_{H} q_{L}-\alpha \leq 0 \\
& (1-\delta)\left(x-\theta_{L} q_{L}-\alpha\right)=(1-\delta) \Delta \theta q_{H}+\delta \min \Phi(\tilde{p}) .
\end{aligned}
$$

(v) If $\min \Phi(p)<\Delta \theta q_{H}^{*}$ and $v \in\left[\min \Phi(p), \Delta \theta q_{H}^{*}\right]$, there exists $p^{\prime} \in[0, p]$ such that $v \in \Phi\left(p^{\prime}\right)$.

The proof of Lemma 8 is provided in the Supplemental Material.

We are now ready to conclude the proof of Proposition 1. First, we consider generic values of $\delta \leq \hat{\delta}$ and use the pair $(V(\cdot), \Phi(\cdot))$ defined in Lemma 8 to construct the equilibrium strategies. We assume that $\underline{p}<\bar{p}$ (the case $\underline{p}=\bar{p}=\hat{p}$ is discussed below together with the case $\delta>\hat{\delta}$ ). 
For every $p \in[0,1]$, we construct a set of menus $\mathbf{m}(p)$.

If $p<\underline{p}$, the set $\mathbf{m}(p)$ contains only the menu $m(p)=\left\{\left(\theta_{H} q_{H}^{*}+\alpha, q_{H}^{*}\right)\right\}$. If $p>\bar{p}$, $\mathbf{m}(p)$ contains only the menu $m(p)=\left\{\left(\theta_{L} q_{L}^{*}+\alpha, q_{L}^{*}\right)\right\}$.

Now consider $p \in[\underline{p}, \bar{p})$ and let $\left(q_{H}(p), q_{L}(p), \tilde{p}(p)\right)$ denote the solution to the optimization problem (18) such that

$$
(1-\delta) \Delta \theta q_{H}(p)+\delta \min \Phi(\tilde{p}(p))=\min \Phi(p) .
$$

We let $m(p)$ denote the menus that contain the contracts $\left(x_{H}(p), q_{H}(p)\right)$ and $\left(x_{L}(p), q_{L}(p)\right)$, where the payments $x_{H}(p)$ and $x_{L}(p)$ are given by

$$
\begin{aligned}
& x_{H}(p)=\theta_{H} q_{H}(p)+\alpha \\
& x_{L}(p)=\theta_{L} q_{L}(p)+\alpha+\Delta \theta q_{H}(p)+\frac{\delta}{1-\delta} \min \Phi(\tilde{p}(p)) .
\end{aligned}
$$

If $\max \Phi(p)=\min \Phi(p)$, then $\mathbf{m}(p)=\{m(p)\}$. If $\max \Phi(p)>\min \Phi(p)$ and $p \in(\underline{p}, \bar{p})$, then we let $\left(q_{H}^{\prime}(p), q_{L}^{\prime}(p), \tilde{p}^{\prime}(p)\right)$ denote the solution to the optimization problem (18) such that

$$
(1-\delta) \Delta \theta q_{H}^{\prime}(p)+\delta \min \Phi\left(\tilde{p}^{\prime}(p)\right)=\max \Phi(p) .
$$

We also let $m^{\prime}(p)$ denote the menus that contain the contracts $\left(x_{H}^{\prime}(p), q_{H}^{\prime}(p)\right)$ and $\left(x_{L}^{\prime}(p), q_{L}^{\prime}(p)\right)$, where the payments $x_{H}^{\prime}(p)$ and $x_{L}^{\prime}(p)$ are given by

$$
\begin{aligned}
& x_{H}^{\prime}(p)=\theta_{H} q_{H}^{\prime}(p)+\alpha \\
& x_{L}^{\prime}(p)=\theta_{L} q_{L}^{\prime}(p)+\alpha+\Delta \theta q_{H}^{\prime}(p)+\frac{\delta}{1-\delta} \min \Phi\left(\tilde{p}^{\prime}(p)\right) .
\end{aligned}
$$

In this case, we set $\mathbf{m}(p)=\left\{m(p), m^{\prime}(p)\right\}$.

If $\max \Phi(\underline{p})>\min \Phi(\underline{p})$, then we set $m^{\prime}(\underline{p})=\left\{\left(\theta_{H} q_{H}^{*}+\alpha, q_{H}^{*}\right)\right\}$ and $\mathbf{m}(\underline{p})=\{m(\underline{p})$, $\left.m^{\prime}(\underline{p})\right\}$.

Finally, we consider $\bar{p}$ and set $m(\bar{p})=\left\{\left(\theta_{L} q_{L}^{*}+\alpha, q_{L}^{*}\right)\right\}$. If $\max \Phi(\bar{p})=\min \Phi(\bar{p})$, then $\mathbf{m}(\bar{p})=\{m(\bar{p})\}$; otherwise, we set $m^{\prime}(\bar{p})=\left\{\left(x_{H}^{\prime}(\bar{p}), q_{H}^{\prime}(\bar{p})\right),\left(x_{L}^{\prime}(\bar{p}), q_{L}^{\prime}(\bar{p})\right)\right\}$ (see (19) and (20)) and $\mathbf{m}(\bar{p})=\left\{m(\bar{p}), m^{\prime}(\bar{p})\right\}$.

The equilibrium strategies are described in terms of the state that consists of a belief $p \in[0,1]$ and a continuation payoff $v \in \Phi(p)$. The initial state is $\left(p_{0}, \min \Phi\left(p_{0}\right)\right)$, where $p_{0}$ is the prior.

Consider an arbitrary public history $h^{t}$ and suppose the state is $(p, v)$. In equilibrium, if $v=\min \Phi(p)$, then the firm offers the menu $m(p)$. Alternatively, if $v>\min \Phi(p)$, then the firm randomizes between the two menus in $\mathbf{m}(p)$ and proposes $m(p)$ with probability $\beta$ defined by

$$
\beta \min \Phi(p)+(1-\beta) \max \Phi(p)=v .
$$

We now turn to the worker's strategy. Consider a public history $h^{t}$ in which the firm's belief $\mu\left(h^{t}\right)$ is equal to $p$. Let $m=\left(\left(x_{1}, q_{1}\right), \ldots,\left(x_{k}, q_{k}\right)\right)$ denote the menu offered by the 
firm and, for $i \in\{H, L\}$, define

$$
\left(\bar{x}_{i}, \bar{q}_{i}\right):=\underset{j=1, \ldots, k}{\arg \max } x_{j}-\theta_{i} q_{j}-\alpha .
$$

If $\bar{x}_{L}-\theta_{L} \bar{q}_{L}-\alpha<0$, then both types reject all the contracts in the menu and quit the relationship. Furthermore, if the worker accepts a contract, then the firm's belief is equal to 1 (in other words, the new state is $(1,0)$ ).

Suppose that $\bar{x}_{L}-\theta_{L} \bar{q}_{L}-\alpha \geq 0$ and $\bar{x}_{H}-\theta_{H} \bar{q}_{H}-\alpha<0$. In this case, the low type picks the contract $\left(\bar{x}_{L}, \bar{q}_{L}\right)$, while the high type quits the relationship. Again, if the worker accepts a contract, the firm's belief is equal to 1 .

We now turn to the case $\bar{x}_{H}-\theta_{H} \bar{q}_{H}-\alpha \geq 0$ and distinguish among three different possibilities. First, assume that the contracts $\left(\bar{x}_{L}, \bar{q}_{L}\right)$ and $\left(\bar{x}_{H}, \bar{q}_{H}\right)$ are such that

$$
(1-\delta)\left(\bar{x}_{L}-\theta_{L} \bar{q}_{L}-\alpha\right) \leq(1-\delta)\left(\bar{x}_{H}-\theta_{L} \bar{q}_{H}-\alpha\right)+\delta \min \Phi(p) .
$$

In this case, both types accept the contract $\left(\bar{x}_{H}, \bar{q}_{H}\right)$ and the firm's belief remains unchanged. If the worker accepts any other contract, the firm's belief will jump to 1 .

Second, if

$$
(1-\delta)\left(\bar{x}_{L}-\theta_{L} \bar{q}_{L}-\alpha\right) \geq(1-\delta)\left(\bar{x}_{H}-\theta_{L} \bar{q}_{H}-\alpha\right)+\delta \Delta \theta q_{H}^{*},
$$

then type $i \in\{H, L\}$ accepts the contract $\left(\bar{x}_{i}, \bar{q}_{i}\right)$. The firm's belief will become 0 if the worker accepts the contract $\left(\bar{x}_{H}, \bar{q}_{H}\right)$ and become 1 if the worker accepts any other contract.

Finally, assume that

$$
\frac{(1-\delta)\left[\left(\bar{x}_{L}-\theta_{L} \bar{q}_{L}\right)-\left(\bar{x}_{H}-\theta_{L} \bar{q}_{H}\right)\right]}{\delta} \in\left(\min \Phi(p), \Delta \theta q_{H}^{*}\right)
$$

and, thus,

$$
(1-\delta)\left(\bar{x}_{L}-\theta_{L} \bar{q}_{L}-\alpha\right)=(1-\delta)\left(\bar{x}_{H}-\theta_{L} \bar{q}_{H}-\alpha\right)+\delta\left[\tilde{\beta} \min \Phi\left(p^{\prime}\right)+(1-\tilde{\beta}) \max \Phi\left(p^{\prime}\right)\right]
$$

for some $p^{\prime} \leq p$ and some $\tilde{\beta} \in[0,1]$. In this case, the high type accepts the contract $\left(\bar{x}_{H}, \bar{q}_{H}\right)$, while the low type chooses the contract $\left(\bar{x}_{H}, \bar{q}_{H}\right)$ with probability $\frac{p^{\prime}}{1-p^{\prime}} \frac{1-p}{p}$ and chooses the contract $\left(\bar{x}_{L}, \bar{q}_{L}\right)$ with probability $1-\frac{p^{\prime}}{1-p^{\prime}} \frac{1-p}{p}$. Following the acceptance of the contract $\left(\bar{x}_{H}, \bar{q}_{H}\right)$, the new state will be $\left(p^{\prime}, \tilde{\beta} \min \Phi\left(p^{\prime}\right)+(1-\tilde{\beta}) \max \Phi\left(p^{\prime}\right)\right)$. If the worker accepts the contract $\left(\bar{x}_{L}, \bar{q}_{L}\right)$ or any other contract, the firm's belief will be equal to 1.

The above strategy profile, together with the firm's belief, constitutes a PBE. The sequential rationality of the firm's strategy follows from the construction of the pair $(V, \Phi)$. In equilibrium, the high type behaves myopically and maximizes his period- $t$ payoff at any history $h^{t}$. This behavior is indeed optimal since the high type's continuation payoff (computed at the beginning of period $t+1$ ) is equal to 0 after any public history. Finally, notice that when the low type randomizes, all the contracts in the strategy's support yield the same expected payoff (and this is greater than the payoff of any other contract). 
We now briefly turn to the case $\delta>\hat{\delta}$ and the case $p=\bar{p}=\hat{p}$ (when $\delta \leq \hat{\delta}$ ). Recall the definitions of $V$ and $\Phi$ in (16) and (17), respectively. For every belief $p$, we define the set of menus $\mathbf{m}(p)$ as follows. If $p<\hat{p}$, the set $\mathbf{m}(p)$ contains only the menu $m(p)=$ $\left\{\left(\theta_{H} q_{H}^{*}+\alpha, q_{H}^{*}\right)\right\}$. If $p>\hat{p}, \mathbf{m}(p)$ contains only the menu $m(p)=\left\{\left(\theta_{L} q_{L}^{*}+\alpha, q_{L}^{*}\right)\right\}$. Finally, the set $\mathbf{m}(\hat{p})$ contains both the menu $m(\hat{p})=\left\{\left(\theta_{L} q_{L}^{*}+\alpha, q_{L}^{*}\right)\right\}$ and the menu $m^{\prime}(\hat{p})=\left\{\left(\theta_{H} q_{H}^{*}+\alpha, q_{H}^{*}\right)\right\}$. The equilibrium strategies and beliefs are defined similarly to the case $\underline{p}<\bar{p}$ above and we omit the details.

\section{Appendix B}

Proof of Lemma 4. Fix a PBE $(\sigma, \mu)$ and a history $h^{t}\left(\mu\left(h^{t}\right)<p\right)$ at which the firm offers a menu $m_{t}$ with the properties described in the statement of the lemma. First, notice that if the high type rejects all the contracts in $m_{t}$ with probability 1 (i.e., the high type quits), then the high type's length of the relationship, the firm's payoff (conditional on the high type), and the low type's payoff are all equal to 0 (if the low type's payoff is strictly positive, the firm's payoff would fall below $\left.\mu\left(h^{t}\right) \pi_{L}\left(q_{L}^{*}\right)\right)$.

Consider now the case in which the high type accepts a contract in $m_{t}$, say $\left(x_{t}^{H}, q_{t}^{H}\right)$, with positive probability. We let $h_{H}^{t+1}$ denote the history $\left(h^{t}, m_{t},\left(x_{t}^{H}, q_{t}^{H}\right)\right)$. We also let $h_{L}^{t+1}$ denote the history $\left(h^{t}, m_{t},\left(x_{t}^{L}, q_{t}^{L}\right)\right)$.

The fact that type $i \in\{H, L\}$ accepts with positive probability the contract $\left(x_{t}^{i}, q_{t}^{i}\right)$ implies that

$$
(1-\delta)\left(x_{t}^{H}-\theta_{H} q_{t}^{H}-\alpha\right)+\delta W_{H}\left(h_{H}^{t+1}\right) \geq(1-\delta)\left(x_{t}^{L}-\theta_{H} q_{t}^{L}-\alpha\right)+\delta W_{H}\left(h_{L}^{t+1}\right)
$$

and

$$
(1-\delta)\left(x_{t}^{L}-\theta_{L} q_{t}^{L}-\alpha\right)+\delta W_{L}\left(h_{L}^{t+1}\right) \geq(1-\delta)\left(x_{t}^{H}-\theta_{L} q_{t}^{H}-\alpha\right)+\delta W_{L}\left(h_{H}^{t+1}\right) .
$$

We add the two incentive compatibility constraints and obtain

$$
(1-\delta) \Delta \theta\left(q_{t}^{L}-q_{t}^{H}\right)+\delta\left(W_{L}\left(h_{L}^{t+1}\right)-W_{H}\left(h_{L}^{t+1}\right)\right) \geq \delta\left(W_{L}\left(h_{H}^{t+1}\right)-W_{H}\left(h_{H}^{t+1}\right)\right) .
$$

Recall that $\mu\left(h_{L}^{t+1}\right) \geq p$ and that $[p, 1]$ is a firing region. Therefore, there exist $\bar{K}$ and $\bar{\delta}<1$ such that $W_{L}\left(h_{L}^{t+1}\right) \leq \bar{K}(1-\delta)$ for $\delta>\bar{\delta}$. Of course, $W_{H}\left(h_{L}^{t+1}\right) \geq 0$. This, together with the above inequality, implies

$$
(1-\delta) \Delta \theta\left(q_{t}^{L}-q_{t}^{H}\right)+\bar{K}(1-\delta) \geq \delta\left(W_{L}\left(h_{H}^{t+1}\right)-W_{H}\left(h_{H}^{t+1}\right)\right) .
$$

We now let $\mathcal{D}_{H}\left(h_{H}^{t+1}\right):=\mathbb{E}_{(\sigma, \mu)}\left[(1-\delta) \sum_{\tau=t+1}^{\mathbb{T}-1} \delta^{\tau-t-1} \mid h_{H}^{t+1}, H\right]$ denote the expected discounted time, computed at $h_{H}^{t+1}$, until the high type quits. Our next goal is to provide an upper bound to $\mathcal{D}_{H}\left(h_{H}^{t+1}\right)$. Thus, without loss, assume that $\mathcal{D}_{H}\left(h_{H}^{t+1}\right)$ is strictly positive. We let

$$
\mathcal{Q}_{t+1}=\frac{\mathbb{E}_{(\sigma, \mu)}\left[(1-\delta) \sum_{\tau=t+1}^{\mathbb{T}-1} \delta^{\tau-t-1} q_{\tau} \mid h_{H}^{t+1}, H\right]}{\mathcal{D}_{H}\left(h_{H}^{t+1}\right)}
$$


denote the expected discounted total quality provided by the high type at the history $h_{H}^{t+1}$.

Using Jensen's inequality (recall that the function $\pi(\cdot)$ is concave), we can bound the firm's continuation payoff (conditional on type $H$ ) as

$$
\begin{aligned}
V_{F}\left(h_{H}^{t+1} ; H\right) & \leq \mathbb{E}_{(\sigma, \mu)}\left[(1-\delta) \sum_{\tau=t+1}^{\mathbb{T}-1} \delta^{\tau-t-1} \pi\left(q_{\tau}\right) \mid h_{H}^{t+1}, H\right] \\
& \leq \mathbb{E}_{(\sigma, \mu)}\left[(1-\delta) \sum_{\tau=t+1}^{\mathbb{T}-1} \delta^{\tau-t-1} \pi\left(\mathcal{Q}_{t+1}\right) \mid h_{H}^{t+1}, H\right]=\mathcal{D}_{H}\left(h_{H}^{t+1}\right) \pi\left(\mathcal{Q}_{t+1}\right) .
\end{aligned}
$$

Let $\breve{q}_{H} \in\left(0, q_{H}^{*}\right)$ be such that $\pi_{H}\left(\breve{q}_{H}\right)=0$ and notice that $\pi_{H}(q)<0$ for every $q<\breve{q}_{H}$. This implies that $\mathcal{Q}_{t+1} \geq \breve{q}_{H}$. In fact, if the last inequality is violated, then $V_{F}\left(h_{H}^{t+1} ; H\right)$ is strictly negative and $V_{F}\left(h_{H}^{t+1}\right)$ is strictly less than $\mu\left(h_{H}^{t+1}\right) \pi_{L}\left(q_{L}^{*}\right)$, contradicting Lemma 1. Notice that one strategy available to the low type is to imitate the high type's behavior (in every period). Therefore, we conclude that

$$
W_{L}\left(h_{H}^{t+1}\right)-W_{H}\left(h_{H}^{t+1}\right) \geq \Delta \theta \mathcal{D}_{H}\left(h_{H}^{t+1}\right) \mathcal{Q}_{t+1} \geq \Delta \theta \mathcal{D}_{H}\left(h_{H}^{t+1}\right) \breve{q}_{H} .
$$

Combining the inequality above with inequality (21), we obtain

$$
\mathcal{D}_{H}\left(h_{H}^{t+1}\right) \leq \frac{(1-\delta)\left(q_{t}^{L}-q_{t}^{H}\right)}{\delta \breve{q}_{H}}+\frac{\bar{K}(1-\delta)}{\delta \Delta \theta \breve{q}_{H}} \leq \frac{(1-\delta)}{\delta \breve{q}_{H}}\left(1+\frac{\bar{K}}{\Delta \theta}\right) .
$$

Hence, for $\delta>\frac{1}{2}$, we have

$$
\mathcal{D}_{H}\left(h_{H}^{t+1}\right) \leq \frac{2(1-\delta)}{\breve{q}_{H}}\left(1+\frac{\bar{K}}{\Delta \theta}\right)
$$

This, in turn, implies that (for $\delta>\frac{1}{2}$ )

$$
\begin{aligned}
\mathbb{E}_{(\sigma, \mu)}\left[(1-\delta) \sum_{\tau=t}^{\mathbb{T}-1} \delta^{\tau-t} \mid h^{t}, m_{t}, H\right] & \leq(1-\delta)+\mathcal{D}_{H}\left(h_{H}^{t+1}\right) \\
& \leq\left(1+\frac{2}{\breve{q}_{H}}+\frac{2 \bar{K}}{\Delta \theta \breve{q}_{H}}\right)(1-\delta):=\tilde{K}(1-\delta)
\end{aligned}
$$

and establishes part (i).

To verify property (ii), notice that the inequality above implies that the firm's continuation payoff $V_{F}\left(h^{t}, m_{t} ;(\sigma, \mu), H\right)$ is bounded above by $v(1) \tilde{K}(1-\delta)$.

Finally, we turn to property (iii). The analysis above implies that

$$
V_{F}\left(h^{t}, m_{t}\right) \leq \mu\left(h^{t}\right)\left[\pi_{L}\left(q_{L}^{*}\right)-W_{L}\left(h^{t}, m_{t}\right)\right]+v(1) \tilde{K}(1-\delta) .
$$

Let $\delta^{\prime}$ be such that

$$
v(1) \tilde{K}\left(1-\delta^{\prime}\right)=\frac{\pi_{H}\left(q_{H}^{*}\right)}{4}
$$


and notice that for $\delta \geq \tilde{\delta}=\max \left\{\delta^{\prime}, \bar{\delta}, \frac{1}{2}\right\}$ and $\mu\left(h^{t}\right) \leq \frac{\pi_{H}\left(q_{H}^{*}\right)}{2 \pi_{L}\left(q_{L}^{*}\right)}$,

$$
\mu\left(h^{t}\right) \pi_{L}\left(q_{L}^{*}\right)+v(1) \tilde{K}(1-\delta) \leq \frac{3}{4} \pi_{H}\left(q_{H}^{*}\right) .
$$

It follows that if the firm offers the menu $m_{t}$ at the history $h^{t}$ and $\delta \geq \tilde{\delta}$, then $\mu\left(h^{t}\right)>\frac{\pi_{H}\left(q_{H}^{*}\right)}{2 \pi_{L}\left(q_{L}^{*}\right)}$. Finally, recall that $V_{F}\left(h^{t}, m_{t}\right)$ is bounded below by $\mu\left(h^{t}\right) \pi_{L}\left(q_{L}^{*}\right)$. This and inequality (22) imply

$$
W_{L}\left(h^{t}, m_{t}\right) \leq \frac{v(1)}{\mu\left(h^{t}\right)} \tilde{K}(1-\delta) \leq \frac{2 \pi_{L}\left(q_{L}^{*}\right) v(1) \tilde{K}}{\pi_{H}\left(q_{H}^{*}\right)}(1-\delta) .
$$

This shows that there exists $K>0$ that satisfies the three properties in Lemma 4 .

Derivation of the inequalities in (3). First, notice that $W_{H}\left(h^{0}\right)=0$ implies that the menu $m_{0}$ offered at $h^{0}$ yields a continuation payoff of 0 to the high type. Let $m_{0}=$ $\left(\left(x_{0}^{1}, q_{0}^{1}\right), \ldots,\left(x_{0}^{k}, q_{0}^{k}\right)\right)$ be the menu offered at $h^{0}$. For $i \in\{H, L\}$, let $m_{0}^{i}$ denote the set of contracts in $m_{0}$ accepted with positive probability by the type $i$. If there exists a con$\operatorname{tract}\left(x_{0}^{j}, q_{0}^{j}\right)$ in $m_{0}^{L} \backslash m_{0}^{H}$, then $\tilde{\mathbb{T}}$ coincides with 0 (in fact, the firm's belief jumps to 1 if the worker accepts the contract $\left.\left(x_{0}^{j}, q_{0}^{j}\right)\right)$ and there is nothing to prove. Therefore, assume that $m_{0}^{L} \subseteq m_{0}^{H}$ and recall that $m_{0}^{L}$ is nonempty.

For every contract $\left(x_{0}^{j}, q_{0}^{j}\right)$ in $m_{0}^{H}$, we let $h_{j}^{1}=\left(h^{0}, m_{0},\left(x_{0}^{j}, q_{0}^{j}\right)\right)$ denote the history in which the worker accepts the contract $\left(x_{0}^{j}, q_{0}^{j}\right)$ in period 0 and we recall that $W_{H}\left(h_{j}^{1}\right) \geq$ 0 represents the high type worker's payoff at $h_{j}^{1}$. For every contract $\left(x_{0}^{j}, q_{0}^{j}\right) \in m_{0}^{H}$, we replace the payment $x_{0}^{j}$ with the payment

$$
\tilde{x}_{0}^{j}=x_{0}^{j}+\frac{\delta}{1-\delta} W_{H}\left(h_{j}^{1}\right),
$$

which clearly implies $\tilde{x}_{0}^{j}=\theta_{H} q_{0}^{j}+\alpha$.

To keep the parties' payoffs unchanged, we also modify the payments in period 1 . In particular, for every $\left(x_{0}^{j}, q_{0}^{j}\right) \in m_{0}^{H}$, consider the history $h_{j}^{1}$. Let $m_{1}$ denote a menu offered at $h_{j}^{1}$ with positive probability. We subtract $\frac{1}{1-\delta} W_{H}\left(h_{j}^{1}, m_{1}\right)$, the high type's continuation payoff at the moment that the menu $m_{1}$ is offered, from the payment of every contract in $m_{1}$. Notice that this yields to the high type a continuation payoff (evaluated at the beginning of period 1) equal to 0 .

We recursively apply the procedure outlined above to periods $t=1, \ldots, \tilde{\mathbb{T}}$ (i.e., we increase the payments in period $t$ and, at the same time, decrease the payments in period $t+1)$. By construction, every contract $\left(x_{t}, q_{t}\right)$ accepted with positive probability by the high type in period $t=0, \ldots, \tilde{\mathbb{T}}-1$ is replaced with the contract $\left(\theta_{H} q_{t}+\alpha, q_{t}\right)$, while the payments in every menu offered in period $\tilde{\mathbb{T}}$ are uniformly decreased by the high type's continuation payoff. Finally, in every period $t=0, \ldots, \tilde{\mathbb{T}}-1$, the set of contracts accepted by the low type is contained in the set of contracts accepted by the high type (this follows from the definition of $\tilde{\mathbb{T}}$ ). 
The change in the timing of the transfers yields

$$
\begin{aligned}
& V_{F}\left(h^{0}\right)=\mathbb{E}_{(\sigma, \mu)}\left[(1-\delta) \sum_{t=0}^{\tilde{\mathbb{T}}-1} \delta^{t} \pi_{H}\left(q_{t}\right)+\mathbb{I}_{\{\tilde{\mathbb{T}}<\infty\}} \delta^{\tilde{\mathbb{T}}}\left(V_{F}\left(h^{\tilde{\mathbb{T}}}, m_{\tilde{\mathbb{T}}}\right)+W_{H}\left(h^{\tilde{\mathbb{T}}}, m_{\tilde{\mathbb{T}}}\right)\right) \mid h^{0}\right] \\
& W_{L}\left(h^{0}\right)=\mathbb{E}_{(\sigma, \mu)}\left[(1-\delta) \sum_{t=0}^{\tilde{\mathbb{T}}-1} \delta^{t} \Delta \theta q_{t}+\mathbb{I}_{\{\tilde{\mathbb{T}}<\infty\}} \delta^{\tilde{\mathbb{T}}}\left(W_{L}\left(h^{\tilde{\mathbb{T}}}, m_{\tilde{\mathbb{T}}}\right)-W_{H}\left(h^{\tilde{\mathbb{T}}}, m_{\tilde{\mathbb{T}}}\right)\right) \mid h^{0}, L\right] \\
& W_{L H}\left(h^{0}\right) \geq \mathbb{E}_{(\sigma, \mu)}\left[\sum_{t=0}^{\tilde{\mathbb{T}}-1} \delta^{t} \Delta \theta q_{t}-\mathbb{I}_{\{\tilde{\mathbb{T}}<\infty\}} \delta^{\tilde{\mathbb{T}}} W_{H}\left(h^{\tilde{\mathbb{T}}}, m_{\tilde{\mathbb{T}}}\right) \mid h^{0}, H\right] .
\end{aligned}
$$

The payoff $W_{H}\left(h^{\tilde{\mathbb{T}}}, m_{\tilde{\mathbb{T}}}\right)$ is bounded above by $W_{L}\left(h^{\tilde{\mathbb{T}}}, m_{\tilde{\mathbb{T}}}\right)$. Therefore, it follows from Lemma 4 and from the fact that $[p, 1]$ is a firing region that there exist $K$ and $\bar{\delta}<1$ such that for any $\delta>\bar{\delta}$ and for any $\operatorname{PBE}(\sigma, \mu)$, the payoffs $V_{F}\left(h^{0}\right), W_{L}\left(h^{0}\right)$, and $W_{L H}\left(h^{0}\right)$ satisfy the inequalities in (3).

Proof of Claim 1. First, assume that $\tilde{q}_{0} \leq \frac{\breve{q}_{H}}{2}$ and notice that $\pi_{H}\left(\tilde{q}_{0}\right)<\pi_{H}\left(\frac{\breve{q}_{H}}{2}\right)<0$ (recall that $\breve{q}_{H} \in\left(0, q_{H}^{*}\right)$ satisfies $\left.\pi_{H}\left(\breve{q}_{H}\right)=0\right)$. Also notice that $\hat{p}<p_{0}<p$. If inequality (8) is satisfied, then we have

$$
\begin{aligned}
0 & \leq\left(1-\frac{p_{0}}{p}\right) \Upsilon_{0} \pi_{H}\left(\tilde{q}_{0}\right)+\frac{p_{0}}{p}\left[\Upsilon_{p} \pi_{H}\left(\tilde{q}_{p}\right)+\left(1-\Upsilon_{p}\right) p \pi_{L}\left(q_{L}^{*}\right)\right]+K(1-\delta)-p_{0} \pi_{L}\left(q_{L}^{*}\right) \\
& \leq\left(1-\frac{p_{0}}{p}\right) \Upsilon_{0} \pi_{H}\left(\frac{\breve{q}_{H}}{2}\right)+\frac{p_{0}}{p} \Upsilon_{p}\left[\pi_{H}\left(q_{H}^{*}\right)-p \pi_{L}\left(q_{L}^{*}\right)\right]+K(1-\delta) \\
& \leq\left(1-\frac{p_{0}}{p}\right) \Upsilon_{0} \pi_{H}\left(\frac{\breve{q}_{H}}{2}\right)+K(1-\delta) .
\end{aligned}
$$

Putting this and $p_{0}<p^{C}$ together, we obtain

$$
\left(\frac{1}{1-p_{0}}\right)\left(1-\frac{p_{0}}{p}\right) \Upsilon_{0} \leq-\frac{K(1-\delta)}{\left(1-p^{C}\right) \pi_{H}\left(\frac{\breve{q}_{H}}{2}\right)}
$$

Similarly, we obtain

$$
\begin{aligned}
0 & \leq\left(1-\frac{p_{0}}{p}\right) \Upsilon_{0} \pi_{H}\left(\tilde{q}_{0}\right)+\frac{p_{0}}{p}\left[\Upsilon_{p} \pi_{H}\left(\tilde{q}_{p}\right)+\left(1-\Upsilon_{p}\right) p \pi_{L}\left(q_{L}^{*}\right)\right]+K(1-\delta)-p_{0} \pi_{L}\left(q_{L}^{*}\right) \\
& \leq\left(1-\frac{p_{0}}{p}\right) \Upsilon_{0} \pi_{H}\left(\tilde{q}_{0}\right)+\frac{p_{0}}{p} \Upsilon_{p}\left[\pi_{H}\left(q_{H}^{*}\right)-p \pi_{L}\left(q_{L}^{*}\right)\right]+K(1-\delta) \\
& \leq \frac{p_{0}}{p} \Upsilon_{p}\left[\pi_{H}\left(q_{H}^{*}\right)-p \pi_{L}\left(q_{L}^{*}\right)\right]+K(1-\delta) \\
& \leq\left(1-\frac{f(p)}{2 p}\right) \Upsilon_{p}\left[\pi_{H}\left(q_{H}^{*}\right)-p \pi_{L}\left(q_{L}^{*}\right)\right]+K(1-\delta)
\end{aligned}
$$

where the last inequality follows from $p_{0} \in\left[p-\frac{f(p)}{2}, p\right]$ and $\pi_{H}\left(q_{H}^{*}\right)-p \pi_{L}\left(q_{L}^{*}\right)<0$. 
Hence, we have

$$
\left(\frac{1-p}{1-p_{0}}\right)\left(\frac{p_{0}}{p}\right) \Upsilon_{p} \leq \Upsilon_{p} \leq \frac{K(1-\delta)}{\left(1-\frac{f(p)}{2 p}\right)\left[p \pi_{L}\left(q_{L}^{*}\right)-\pi_{H}\left(q_{H}^{*}\right)\right]}
$$

For the case $\tilde{q}_{0} \leq \frac{\breve{q}_{H}}{2}$, inequalities (23) and (24) imply the result.

We now move to the case $\tilde{q}_{0}>\frac{\breve{q}_{H}}{2}$. It follows from the concavity of $\pi_{H}(\cdot)$ that $\pi_{H}\left(\tilde{q}_{0}\right) \leq \pi_{H}(0)+\pi_{H}^{\prime}(0) \tilde{q}_{0} \leq \pi_{H}^{\prime}(0) \tilde{q}_{0}$. Also, $\pi_{H}\left(\tilde{q}_{p}\right)<\pi_{H}\left(q_{H}^{*}\right)$ for any $\tilde{q}_{p} \neq q_{H}^{*}$. Thus, we have

$$
\begin{aligned}
& \left(1-\frac{p_{0}}{p}\right) \Upsilon_{0} \pi_{H}\left(\tilde{q}_{0}\right)+\frac{p_{0}}{p}\left[\Upsilon_{p} \pi_{H}\left(\tilde{q}_{p}\right)+\left(1-\Upsilon_{p}\right) p \pi_{L}\left(q_{L}^{*}\right)\right]+K(1-\delta) \\
& \quad \leq\left(1-\frac{p_{0}}{p}\right) \Upsilon_{0} \pi_{H}^{\prime}(0) \tilde{q}_{0}+\frac{p_{0}}{p}\left[\Upsilon_{p} \pi_{H}\left(q_{H}^{*}\right)+\left(1-\Upsilon_{p}\right) p \pi_{L}\left(q_{L}^{*}\right)\right]+K(1-\delta)
\end{aligned}
$$

Suppose that inequality (9) holds. Clearly, the inequality continues to hold if we replace $\tilde{q}_{p}$ with 1 . This allows us to conclude that $\tilde{q}_{0}, \Upsilon_{0}$, and $\Upsilon_{p}$ must satisfy

$$
\Upsilon_{0} \tilde{q}_{0} \leq \Upsilon_{p}+\frac{2 K(1-\delta)}{\Delta \theta\left(\frac{1}{1-p_{0}}\right)\left(1-\frac{p_{0}}{p}\right)}
$$

Combining inequalities (25) and (26), we obtain (recall that $p_{0}>\hat{p}$ )

$$
\begin{aligned}
(1- & \left.\frac{p_{0}}{p}\right) \Upsilon_{0} \pi_{H}\left(\tilde{q}_{0}\right)+\frac{p_{0}}{p}\left[\Upsilon_{p} \pi_{H}\left(\tilde{q}_{p}\right)+\left(1-\Upsilon_{p}\right) p \pi_{L}\left(q_{L}^{*}\right)\right]+K(1-\delta) \\
\leq & \left(1-\frac{p_{0}}{p}\right) \pi_{H}^{\prime}(0) \Upsilon_{p}+\frac{p_{0}}{p}\left[\Upsilon_{p} \pi_{H}\left(q_{H}^{*}\right)+\left(1-\Upsilon_{p}\right) p \pi_{L}\left(q_{L}^{*}\right)\right] \\
& +K\left(1+\frac{2 \pi_{H}^{\prime}(0)(1-\hat{p})}{\Delta \theta}\right)(1-\delta) .
\end{aligned}
$$

We define $K_{1}:=\left(1+\frac{2 \pi_{H}^{\prime}(0)(1-\hat{p})}{\Delta \theta}\right)$. It follows from inequality (8) and the inequality above that

$$
p_{0} \pi_{L}\left(q_{L}^{*}\right) \leq\left(1-\frac{p_{0}}{p}\right) \pi_{H}^{\prime}(0) \Upsilon_{p}+\frac{p_{0}}{p}\left[\Upsilon_{p} \pi_{H}\left(q_{H}^{*}\right)+\left(1-\Upsilon_{p}\right) p \pi_{L}\left(q_{L}^{*}\right)\right]+K_{1}(1-\delta),
$$

which leads to

$$
\begin{aligned}
0 & \leq \Upsilon_{p}\left[\left(1-\frac{p_{0}}{p}\right) \pi_{H}^{\prime}(0)+\frac{p_{0}}{p}\left[\pi_{H}\left(q_{H}^{*}\right)-p \pi_{L}\left(q_{L}^{*}\right)\right]\right]+K_{1}(1-\delta) \\
& \leq \Upsilon_{p}\left[\left(1-\frac{p-\frac{f(p)}{2}}{p}\right) \pi_{H}^{\prime}(0)+\frac{p-\frac{f(p)}{2}}{p}\left[\pi_{H}\left(q_{H}^{*}\right)-p \pi_{L}\left(q_{L}^{*}\right)\right]+K_{1}(1-\delta) .\right.
\end{aligned}
$$


The second inequality holds because the expression $\left(1-\frac{p_{0}}{p}\right) \pi_{H}^{\prime}(0)+\frac{p_{0}}{p}\left[\pi_{H}\left(q_{H}^{*}\right)-\right.$ $\left.p \pi_{L}\left(q_{L}^{*}\right)\right]$ is affine in $p_{0}$, is negative for $p_{0}=p$, and equal to 0 for $p_{0}=p-f(p)$ (see the definition of the function $f(\cdot)$ in (2)). Also, recall that $p_{0} \in\left[p-\frac{f(p)}{2}, p\right]$.

From inequality (27), we obtain

$$
\Upsilon_{p} \leq \frac{K_{1}}{\left(p-\frac{f(p)}{2}\right) \pi_{L}\left(q_{L}^{*}\right)-\left(\frac{f(p)}{2 p}\right) \pi_{H}^{\prime}(0)-\left(1-\frac{f(p)}{2 p}\right) \pi_{H}\left(q_{H}^{*}\right)}(1-\delta):=K_{2}(1-\delta)
$$

and, thus,

$$
\left(\frac{1-p}{1-p_{0}}\right)\left(\frac{p_{0}}{p}\right) \Upsilon_{p} \leq \Upsilon_{p} \leq K_{2}(1-\delta) .
$$

Finally, using (26) and (28) and $p_{0}<p^{C}$ we have

$$
\begin{aligned}
& \left(\frac{1}{1-p_{0}}\right)\left(1-\frac{p_{0}}{p}\right) \Upsilon_{0} \tilde{q}_{0} \\
& \quad \leq\left(\frac{1}{1-p^{C}}\right)\left(1-\frac{p_{0}}{p}\right) \Upsilon_{p}+\frac{2 K(1-\delta)}{\Delta \theta} \\
& \quad \leq\left(\frac{1}{1-p^{C}}\right) \Upsilon_{p}+\frac{2 K(1-\delta)}{\Delta \theta} \leq\left(\frac{1}{1-p^{C}}\right) K_{2}(1-\delta)+\frac{2 K(1-\delta)}{\Delta \theta} .
\end{aligned}
$$

Recall that $\tilde{q}_{0}>\frac{\breve{q}_{H}}{2}$. It follows from the last inequality that

$$
\left(\frac{1}{1-p_{0}}\right)\left(1-\frac{p_{0}}{p}\right) \Upsilon_{0} \leq\left(\frac{2}{\breve{q}_{H}}\right)\left[\frac{K_{2}}{1-p^{C}}+\frac{2 K}{\Delta \theta}\right](1-\delta),
$$

which coupled with (28) implies the result.

Proof of Lemma 6. Fix a PBE $(\sigma, \mu)$ and a history $h^{t}$ as described in the statement of the lemma. The firm's continuation payoff after offering the menu $m_{t}$ is

$$
V_{F}\left(h^{t}, m_{t}\right)=\left(1-\mu\left(h^{t}\right)\right) V_{F}\left(h^{t}, m_{t} ; H\right)+\mu\left(h^{t}\right) V_{F}\left(h^{t}, m_{t} ; L\right) .
$$

Recall from Proposition 2 that $[\hat{p}+\varepsilon, 1]$ is a firing region. Therefore, it follows from Lemma 4 that there exist $\bar{K}$ and $\bar{\delta}>1-\frac{\varepsilon \pi_{L}\left(q_{L}^{*}\right)}{2 \bar{K}}$ such that $\delta>\bar{\delta}$ implies $V_{F}\left(h^{t}, m_{t} ; H\right) \leq$ $\bar{K}(1-\delta)$. This, in turn, implies

$$
V_{F}\left(h^{t}, m_{t}\right) \leq(\hat{p}-\varepsilon) \pi_{L}\left(q_{L}^{*}\right)+\bar{K}(1-\delta)<\left(\hat{p}-\frac{\varepsilon}{2}\right) \pi_{L}\left(q_{L}^{*}\right)<\pi_{H}\left(q_{H}^{*}\right),
$$

contradicting Lemma 1.

Proof of Proposition 3. We start by proving the first property of a pooling region. Without loss of generality, we establish the property at the initial history $h^{0}$ and restrict attention to equilibria in which (a) the firm's strategy in the first period is pure and (b) the high type's equilibrium payoff is equal to 0 . 
Fix $p<\hat{p}$. By contradiction, suppose that there exists a sequence $\left\{\delta_{n}, p_{0, n}\right.$, $\left.\left(\sigma_{n}, \mu_{n}\right)\right\}_{n=1}^{\infty}$ such that $\delta_{n}$ converges to $1, p_{0, n} \in[0, p],\left(\sigma_{n}, \mu_{n}\right)$ is a PBE of the game with discount factor equal to $\delta_{n}$ and prior equal to $p_{0, n}$, and

$$
\lim _{n \rightarrow \infty} \mathbb{E}_{\left(\sigma_{n}, \mu_{n}\right)}\left[\delta_{n}^{\mathbb{T}}\right]=\xi>0 .
$$

Let $\underline{k}:=\left\lfloor\frac{2}{1-\hat{p}}\right\rfloor$ and, for $k=\underline{k}, \underline{k}+1, \ldots$, let $\tilde{\mathbb{T}}_{k} \leq \infty$ be the random time that stops the play at the first history $\left(h^{\tilde{\mathbb{T}}}, m_{\tilde{\mathbb{T}}}\right)$ at which the menu $m_{\tilde{\mathbb{T}}}$ contains a contract $\left(x_{\tilde{\mathbb{T}}}, q_{\tilde{\mathbb{T}}}\right)$ accepted with positive probability and for which $\mu\left(h^{\tilde{\mathbb{T}}}, m_{\tilde{\mathbb{T}}},\left(x_{\tilde{\mathbb{T}}}, q_{\tilde{\mathbb{T}}}\right)\right) \geq \hat{p}+\frac{1}{k}$. As usual, we set $\tilde{\mathbb{T}}_{k}=\infty$ if the event does not occur in finite time.

It follows from Lemma 4 that for every $k \geq \underline{k}$, there exist $n_{k}^{1} \in \mathbf{N}$ and $K_{k}^{1}$ such that for every $n \geq n_{k}^{1}$, the PBE $\left(\sigma_{n}, \mu_{n}\right)$ satisfies the following property. If the firm offers a menu with a contract that is accepted with positive probability and leads to a belief weakly higher than $\hat{p}+\frac{1}{k}$, then the expected discounted time until the high type quits the relationship is bounded above by $K_{k}^{1}\left(1-\delta_{n}\right)$. Thus, for $n \geq n_{k}^{1}$, we have

$$
\left|\mathbb{E}_{\left(\sigma_{n}, \mu_{n}\right)}\left[\delta_{n}^{\mathbb{T}}\right]-\mathbb{E}_{\left(\sigma_{n}, \mu_{n}\right)}\left[\mathbb{I}_{\left\{\tilde{\mathbb{T}}_{k}<\infty\right\}}\left(1-\mu_{n}\left(h^{\tilde{\mathbb{T}}_{k}}\right)\right) \delta_{n}^{\tilde{\mathbb{T}}_{k}}\right]\right| \leq K_{k}^{1}\left(1-\delta_{n}\right) .
$$

Next, recall that $\left[\hat{p}+\frac{1}{k}\right]$ is a firing region and Lemma 4 (property (ii)) provides an upper bound to the firm's continuation payoff when it offers a menu with a contract that leads to a firing region. Therefore, for every $k \geq \underline{k}$, there exist $n_{k}^{2} \in \mathbf{N}$ and $K_{k}^{2}$ such that for every $n \geq n_{k}^{2}$, the firm's equilibrium payoff is bounded as ${ }^{22}$

$$
\begin{aligned}
V_{F}\left(h^{0} ;\left(\sigma_{n}, \mu_{n}\right)\right) \leq & \mathbb{E}_{\left(\sigma_{n}, \mu_{n}\right)}\left[\mathbb{I}_{\left\{\tilde{\mathbb{T}}_{k}<\infty\right\}}\left[\left(1-\delta_{n}\right) \sum_{t=0}^{\tilde{\mathbb{T}}_{k}-1} \delta_{n}^{t} \pi_{H}\left(q_{t}\right)+\delta_{n}^{\tilde{\mathbb{T}}_{k}} \mu_{n}\left(h^{\tilde{\mathbb{T}}_{k}}\right) \pi_{L}\left(q_{L}^{*}\right)\right]\right. \\
& \left.+\mathbb{I}_{\left\{\tilde{\mathbb{T}}_{k}=\infty\right\}}\left(1-\delta_{n}\right) \sum_{t=0}^{\tilde{\mathbb{T}}_{k}-1} \delta_{n}^{t} \pi_{H}\left(q_{t}\right)\right]+K_{k}^{2}\left(1-\delta_{n}\right) .
\end{aligned}
$$

Notice that when $\tilde{\mathbb{T}}_{k}<\infty$, the belief $\mu_{n}\left(h^{\tilde{\mathbb{T}}_{k}}\right)$ is, by definition, lower than $\hat{p}+\frac{1}{k}$ and, therefore, we have

$$
\mu_{n}\left(h^{\tilde{\mathbb{T}}_{k}}\right) \pi_{L}\left(q_{L}^{*}\right)<\left(\hat{p}+\frac{1}{k}\right) \pi_{L}\left(q_{L}^{*}\right)=\pi_{H}\left(q_{H}^{*}\right)+\frac{1}{k} \pi_{L}\left(q_{L}^{*}\right)
$$

Combining the last two inequalities, for every $n \geq n_{k}^{2}$, we obtain

$$
\begin{aligned}
V_{F}\left(h^{0} ;\left(\sigma_{n}, \mu_{n}\right)\right) \leq & \mathbb{E}_{\left(\sigma_{n}, \mu_{n}\right)}\left[\mathbb{I}_{\left\{\tilde{\mathbb{T}}_{k}<\infty\right\}}\left[\left(1-\delta_{n}\right) \sum_{t=0}^{\tilde{\mathbb{T}}_{k}-1} \delta_{n}^{t} \pi_{H}\left(q_{t}\right)+\delta_{n}^{\tilde{\mathbb{T}}_{k}} \pi_{H}\left(q_{H}^{*}\right)\right]\right. \\
& \left.+\mathbb{I}_{\left\{\tilde{\mathbb{T}}_{k}=\infty\right\}}\left(1-\delta_{n}\right) \sum_{t=0}^{\tilde{\mathbb{T}}_{k}-1} \delta_{n}^{t} \pi_{H}\left(q_{t}\right)\right]+K_{k}^{2}\left(1-\delta_{n}\right)+\frac{1}{k} \pi_{L}\left(q_{L}^{*}\right)
\end{aligned}
$$

\footnotetext{
${ }^{22}$ This bound is derived using the same procedure as the one outlined above in the derivation of the inequalities in (3).
} 


$$
\begin{aligned}
= & \pi_{H}\left(q_{H}^{*}\right)-\mathbb{E}_{\left(\sigma_{n}, \mu_{n}\right)}\left[\left(1-\delta_{n}\right) \sum_{t=0}^{\tilde{\mathbb{T}}_{k}-1} \delta_{n}^{t}\left[\pi_{H}\left(q_{H}^{*}\right)-\pi_{H}\left(q_{t}\right)\right]\right] \\
& +K_{k}^{2}\left(1-\delta_{n}\right)+\frac{1}{k} \pi_{L}\left(q_{L}^{*}\right) .
\end{aligned}
$$

This and the fact that the firm's payoff is bounded below by $\pi_{H}\left(q_{H}^{*}\right)$ lead to the result, for every $k \geq \underline{k}$,

$$
\lim \sup _{n \rightarrow \infty} \mathbb{E}_{\left(\sigma_{n}, \mu_{n}\right)}\left[\left(1-\delta_{n}\right) \sum_{t=0}^{\tilde{\mathbb{T}}_{k}-1} \delta_{n}^{t}\left[\pi_{H}\left(q_{H}^{*}\right)-\pi_{H}\left(q_{t}\right)\right]\right] \leq \frac{1}{k} \pi_{L}\left(q_{L}^{*}\right) .
$$

Inequality (31) implies that for every $\eta>0$, there exists $k_{\eta} \in \mathbf{N}$ such that for every $k \geq k_{\eta}$, there exists $\hat{n}_{k} \in \mathbf{N}$ such that for $n \geq \hat{n}_{k}$, we have

$$
\mathbb{E}_{\left(\sigma_{n}, \mu_{n}\right)}\left[\left(1-\delta_{n}\right) \sum_{t=0}^{\tilde{\mathbb{T}}_{k}-1} \delta_{n}^{t}\left|q_{H}^{*}-q_{t}\right|\right] \leq \eta .
$$

Furthermore, $k_{\eta}$ and $\hat{n}_{k}$ are such that for every $k \geq k_{\eta}$ and every $n \geq \hat{n}_{k}$,

$$
\frac{\xi}{1-\hat{p}}-\eta \leq \mathbb{E}_{\left(\sigma_{n}, \mu_{n}\right)}\left[\mathbb{I}_{\left\{\tilde{\mathbb{T}}_{k}<\infty\right\}} \delta_{n}^{\tilde{\mathbb{T}}_{k}}\right] \leq \frac{\xi}{1-\hat{p}}+\eta
$$

The above result is a consequence of equality (29), inequality (30), and Lemma 6.

Fix $\varepsilon \in\left(0, \frac{\Delta \theta q_{H}^{*} \xi}{4(1-\hat{p})\left(1+\Delta \theta q_{H}^{*}\right)}\left(\frac{\hat{p}}{p}-\frac{1-\hat{p}}{1-p}\right)\right)$. Recall that for every $k$, the interval $\left[\hat{p}+\frac{1}{k}, 1\right]$ is a firing region and Lemma 4 (property (iii)) provides an upper bound to the low type's continuation payoff when the firm's menu contains a contract that leads to a firing region. Finally, recall that if a history $h^{t}$ is reached with probability $\operatorname{Pr}\left(h^{t}\right)$ under $\left(\sigma_{n}, \mu_{n}\right)$, then that history is reached with probability $\frac{\mu_{n}\left(h^{t}\right)}{p_{0, n}} \operatorname{Pr}\left(h^{t}\right)$ if the worker behaves according to $\sigma_{n}^{L}$ and is reached with probability $\frac{\left(1-\mu_{n}\left(h^{t}\right)\right)}{\left(1-p_{0, n}\right)} \operatorname{Pr}\left(h^{t}\right)$ if the worker behaves according to $\sigma_{n}^{H}$.

Putting together these observations and the last three inequalities, we conclude that there exist $\tilde{k}$ and $\tilde{n}$ such that for every $n \geq \tilde{n}$, the low type obtains a payoff of at most

$$
\begin{aligned}
\Delta \theta q_{H}^{*}\left(1-\mathbb{E}_{\left(\sigma_{n}, \mu_{n}\right)}\left[\delta_{n}^{\tilde{\mathbb{T}}_{\tilde{k}}} \mid \sigma_{n}^{L}\right]\right)+\varepsilon & \leq \Delta \theta q_{H}^{*}\left(1-\frac{\hat{p}}{p_{0, n}} \frac{\xi}{1-\hat{p}}+\varepsilon\right)+\varepsilon \\
& \leq \Delta \theta q_{H}^{*}\left(1-\frac{\hat{p}}{p} \frac{\xi}{1-\hat{p}}+\varepsilon\right)+\varepsilon
\end{aligned}
$$

when he behaves according to $\sigma_{n}^{L}$ and obtains a payoff of at least

$$
\begin{aligned}
\Delta \theta q_{H}^{*}\left(1-\mathbb{E}_{\left(\sigma_{n}, \mu_{n}\right)}\left[\delta_{n}^{\tilde{\mathbb{T}} \tilde{k}} \mid \sigma_{n}^{H}\right]\right)-\varepsilon & \geq \Delta \theta q_{H}^{*}\left(1-\frac{1-\hat{p}}{1-p_{0, n}} \frac{\xi}{1-\hat{p}}-\varepsilon\right)-\varepsilon \\
& \geq \Delta \theta q_{H}^{*}\left(1-\frac{1-\hat{p}}{1-p} \frac{\xi}{1-\hat{p}}-\varepsilon\right)-\varepsilon
\end{aligned}
$$


when he behaves according to $\sigma_{n}^{H}$. Notice that

$$
\begin{aligned}
& \Delta \theta q_{H}^{*}\left(1-\frac{1-\hat{p}}{1-p} \frac{\xi}{1-\hat{p}}-\varepsilon\right)-\varepsilon-\Delta \theta q_{H}^{*}\left(1-\frac{\hat{p}}{p} \frac{\xi}{1-\hat{p}}+\varepsilon\right)-\varepsilon \\
& =\Delta \theta q_{H}^{*} \frac{\xi}{1-\hat{p}}\left(\frac{\hat{p}}{p}-\frac{1-\hat{p}}{1-p}\right)-2 \varepsilon\left(1+\Delta \theta q_{H}^{*}\right)>0
\end{aligned}
$$

which implies that for $n$ sufficiently large, the low type has an incentive to deviate and follow $\sigma_{n}^{H}$ instead of the equilibrium strategy $\sigma_{n}^{L}$.

We now turn to the second property of a pooling region. It is again without loss of generality to restrict attention to equilibria in which (a) the firm's strategy in the first period is pure and (b) the high type's equilibrium payoff is equal to 0 . The second property follows directly from the first property and inequality (32).

Finally, we establish the third property. Assume, toward a contradiction, that there exists a sequence $\left\{\delta_{n}, p_{0, n},\left(\sigma_{n}, \mu_{n}\right)\right\}_{n=1}^{\infty}$ such that $\delta_{n}$ converges to $1, p_{0, n} \in[0, p],\left(\sigma_{n}, \mu_{n}\right)$ is an arbitrary $\mathrm{PBE}$ of the game with discount factor equal to $\delta_{n}$ and prior equal to $p_{0, n}$, and

$$
\lim _{n \rightarrow \infty} \mathbb{E}_{\left(\sigma_{n}, \mu_{n}\right)}\left[\left(1-\delta_{n}\right) \sum_{t=0}^{\mathbb{T}-1} \delta_{n}^{t}\left(x_{t}-\theta_{H} q_{H}^{*}-\alpha\right) \mid i\right]=\tilde{\xi}>0
$$

for some $i \in\{H, L\}$. Using the first two properties of a pooling region, we conclude that

$$
\lim \sup _{n \rightarrow \infty} V_{F}\left(h^{0} ;\left(\sigma_{n}, \mu_{n}\right)\right) \leq \pi_{H}\left(q_{H}^{*}\right)-\min \left\{p_{0}, 1-p_{0}\right\} \tilde{\xi}<\pi_{H}\left(q_{H}^{*}\right),
$$

which leads to a contradiction and concludes the proof.

\section{REFERENCES}

Acemoglu, Daron, Mikhail Golosov, and Aleh Tsyvinski (2010), "Dynamic Mirrlees taxation under political economy constraints." Review of Economic Studies, 77, 841-881. [586]

Aumann, Robert J. and Michael B. Maschler (1995), Repeated Games With Incomplete Information. MIT Press. [601]

Ausubel, Lawrence M. and Raymond J. Deneckere (1989), "Reputation in bargaining and durable goods monopoly.” Econometrica, 57, 511-531. [586]

Beccuti, Juan and Mark Möller (2018), "Dynamic adverse selection with a patient seller." Journal of Economic Theory, 173, 95-117. [585]

Bhaskar, V. (2014), “The ratchet effect re-examined: A learning perspective.” Working paper, No 9956, CEPR Discussion Papers. [586]

Bhaskar, V. and George J. Mailath (2019), “The curse of long horizons.” Journal of Mathematical Economics, 82, 74-89. [586] 
Carmichael, L. and Bentley MacLeod (2000), "Worker cooperation and the ratchet effect.” Journal of Labor Economics, 18, 1-19. [586]

Farhi, E., C. Sleet, I. Werning, and Sevin Yeltekin (2012), "Non-linear capital taxation without commitment.” Review of Economic Studies, 79, 1469-1493. [586]

Freixas, Xavier, Roger Guesnerie, and Jean Tirole (1985), "Planning under incomplete information and the ratchet effect." Review of Economic Studies, 52, 173-191. [585]

Fudenberg, Drew and Jean Tirole (1991), "Perfect Bayesian equilibrium and sequential equilibrium.” Journal of Economic Theory, 53, 236-260. [589]

Gibbons, Robert (1987), “Piece-rate incentive schemes." Journal of Labor Economics, 5, 413-429. [585]

Halac, Marina (2012), "Relational contracts and the value of relationships." American Economic Review, 102, 750-779. [585]

Hart, Oliver and Jean Tirole (1988), "Contract renegotiation and coasian dynamics." Review of Economic Studies, 55, 509-540. [585]

Kamenica, Emir and Matthew Gentzkow (2011), "Bayesian persuasion." American Economic Review, 101, 2590-2615. [601]

Kanemoto, Yoshitsugu and Bentley MacLeod (1992), "The ratchet effect and the market for secondhand workers." Journal of Labor Economics, 10, 85-98. [586]

Laffont, Jean-Jacques and Jean Tirole (1987), "Comparative statics of the optimal dynamic incentive contract." European Economic Review, 31, 901-926. [585]

Laffont, Jean-Jacques and Jean Tirole (1988), "The dynamics of incentive contracts." Econometrica, 56, 1153-1175. [585]

Laffont, Jean-Jacques and Jean Tirole (1990), "Adverse selection and renegotiation in procurement.” Review of Economic Studies, 57, 597-625. [584, 586]

Laffont, Jean-Jacques and Jean Tirole (1993), A Theory of Incentives in Procurement and Regulation. MIT Press. [590]

Maestri, Lucas (2017), "Dynamic contracting under adverse selection and renegotiation." Journal of Economic Theory, 171, 136-173. [586]

Peski, Marcin (2008), "Repeated games with incomplete information on one side." Theoretical Economics, 3, 29-84. [585]

Scheuer, Florian and Alexander Wolitzky (2016), "Capital taxation under political constraints.” American Economic Review, 106, 2304-2328. [586]

Skreta, Vasiliki (2006), “Sequentially optimal mechanisms.” Review of Economic Studies, 73, 1085-1111. [586]

Skreta, Vasiliki (2015), “Optimal auction design under non-commitment." Journal of Economic Theory, 159, 854-890. [586] 
Strulovici, Bruno (2017), "Contract negotiation and the coase conjecture: A strategic foundation for renegotiation-proof contracts." Econometrica, 85, 585-616. [586]

Co-editor George J. Mailath handled this manuscript.

Manuscript received 23 February, 2016; final version accepted 26 September, 2019; available online 8 October, 2019. 